\title{
Executive Stock Options: Portfolio Effects
}

\author{
Vicky Henderson ${ }^{\dagger}$ \\ Jia Sun ${ }^{\ddagger}$ \\ A. Elizabeth Whalley $\S$ \\ University of Oxford \\ China Credit Rating Co. Ltd \\ Warwick Business School \\ 19th November, 2012
}

\footnotetext{
${ }^{\dagger}$ Oxford-Man Institute, University of Oxford, Eagle House, Walton Well Road, Oxford. OX2 6ED. UK. Email: vicky.henderson@oxford-man.ox.ac.uk

${ }^{\ddagger}$ China Credit Rating Co. Ltd, Ying Tai Mansion. 28 Jin Rong Street, Beijing, Beijing 100032, China. Email: sunjia@chinaratings.com.cn

${ }^{\S}$ Finance Group, Warwick Business School, University of Warwick, Coventry. CV4 7AL. UK. Email: elizabeth.whalley@wbs.ac.uk
} 


\title{
Executive Stock Options: Portfolio Effects
}

\begin{abstract}
Since executives typically receive new grants of stock options (ESOs) each year, longerserving executives often have portfolios of ESOs with differing strikes and maturities. Valuation models for stand-alone ESO grants have shown that trading restrictions, which force executives to bear unhedgeable risk until the options are exercised, induce earlier exercise and hence a lower cost to shareholders than in a risk-neutral setting. However, since unhedgeable risk varies non-linearly with portfolio size and composition, the executive's exercise strategy and thus also the shareholder cost of an option held as part of a portfolio depend also on the remainder of the executive's ESO portfolio. We show that such portfolio effects matter - lowering both the moneyness required for exercise and the shareholder cost of most options in a portfolio. In contrast to a risk-neutral setting, both exercise thresholds and costs depend on an option's position in the optimal exercise order. An option's cost varies with the maturities and, nonlinearly, with the strike prices of other options in the executive's portfolio. The model explains several empirical findings in the literature - which options are attractive to exercise first, how exercise can be induced by a new grant, and the prevalence of early exercise.
\end{abstract}

Keywords: Executive stock options, employee stock options, option portfolios, risk aversion, optimal exercise, American options

JEL Classification Numbers: C61, G11, G13, G30, J33 


\section{Introduction}

Stock options have been an important component of executive compensation packages since the early 1990s. By 2001, stock options accounted for over half of median compensation for CEO's in US S\&P 500 firms. In 2008 stock options still represented $25 \%$ of total pay whilst restricted stock comprised a further 32\% (Conyon et al (2011)). More than half of the S\&P 500 CEO's have received both options and restricted stock annually since 2006. Similarly, Frydman \& Sachs (2010) find options represent around $40 \%$ of total compensation for top executives (2000-2005).

Typically, executives receive a new grant of executive stock options (ESOs) each year, so after several years with a firm, executives can build up a portfolio of options, each with differing strike prices and remaining times to maturity. It is well known that because of restrictions on their ability to hedge these option grants using the firm's shares, the executive's valuation of and optimal exercise threshold for these ESOs can be substantially lower than that in a BlackScholes world, and that as a result the cost of the ESOs to the firm's shareholders can also be considerably less than the options' Black-Scholes values. However, thus far the literature has considered only single grants of options with the same strike price and time to maturity. In this paper we investigate the effects of an executive holding multiple grants of ESOs, each with differing strike price and time to maturity, on the exercise threshold and cost to the shareholders of each option.

Understanding executives' exercise behavior is key to studying their subjective valuation and the cost to shareholders of the options granted. A better knowledge of valuation and costs will assist firms in awarding options more effectively. Estimates of shareholder costs are also required to satisfy accounting regulations in the US and Europe (FASB 123 and IFRS 2). Executives' exercise behavior is also of wider importance in the corporate finance literature, eg. Malmendier and Tate (2005) use option exercise to detect optimism ${ }^{1}$ and Bergstresser and Philippon (2006) interpret option exercise as a signal of private information. In this paper we contribute new findings on exercise behavior, valuation and shareholder costs when the executive holds several option grants. In particular, we answer the question raised by Carpenter, Stanton \& Wallace (2010): "It would be useful to understand which options are most attractive to exercise first" (p $318)$.

We find that the presence of additional options in an executive's ESO portfolio can change the

\footnotetext{
${ }^{1}$ In particular, in constructing their measures of overconfidence they study "late" exercise behavior and calibrate a single threshold for all CEOs which does not take into account the heterogeneity of CEOs option portfolios.
} 
level and shape of a particular option's exercise threshold, even introducing jump discontinuities. This is due to interaction effects between different options in the portfolio and means that thresholds and costs for individual options in a portfolio cannot be calculated on a stand-alone basis, but need to be determined for the portfolio as a whole, and that thresholds and costs for a particular option will vary, depending on the composition of the portfolio it is a part of. Overall, the presence of additional options lowers the exercise threshold for some, if not all, options in the executive's portfolio, including the lowest threshold at which any option is first exercised, and increases the proportional discount of the cost of the portfolio to the shareholders, relative to its Black-Scholes value.

The inability of the executive to hedge her option portfolio using the firm's shares exposes her to risk whilst she continues to hold the options. If she can hedge using the market, the systematic portion of this risk can be removed; however the firm-specific risk of her position remains unhedgeable. Under utility-based valuation, the effective cost of this unhedgeable risk reduces the executive's valuation of her options, but only whilst she continues to hold them: once they have been exercised, the shares acquired on exercise will be sold and the proceeds invested (optimally) in tradeable securities. Her early exercise strategy thus takes account of the reduction in her unhedgeable risk on exercise, and so she exercises at a lower level of moneyness than in a world where risks can be perfectly hedged.

Portfolio effects arise because unhedgeable risk increases non-linearly with portfolio size. For example, the unhedgeable risk associated with a holding of two identical options is four times the unhedgeable risk associated with a single option, and the reduction in unhedgeable risk associated with exercising the first of these two identical options is three times the unhedgeable risk reduction associated with exercising the last. The optimal exercise threshold for an option if it is the first option to be exercised in a portfolio is distinctly lower than the exercise threshold for the same option if it is exercised last (or later in the exercise order). More generally, the optimal exercise threshold for a particular option is lower, the larger the option portfolio remaining on exercise. If the option's position in the optimal exercise order for the portfolio changes, say from penultimate- to last-to-be-exercised, or vice versa, then the unhedgeable risk reduction on exercise also alters instantaneously, so the exercise threshold jumps (up if the new position in the exercise order is later, down if it is earlier).

In a portfolio with multiple options, the exercise threshold for a particular option at any time is never higher than its threshold as a stand-alone option, but it may be lower if the 
option is not last-to-be-exercised. The moneyness at which an option is exercised when it is part of a portfolio depends on the level of the executive's exposure to unhedgeable risk from the portfolio after exercise (the greater the exposure from the portfolio after exercise, the greater the reduction in exposure due to exercise and the lower moneyness at which the option is exercised.) So factors which alter this exposure, most importantly the size, but also the level of moneyness and maturity dates of the remaining portfolio, affect the exercise threshold. All else equal, increasing the size of the portfolio by adding an option decreases the moneyness at which the first option is exercised: this clearly increases the executive's exposure after exercise and the marginal exposure decreases on exercise. Keeping all else fixed, increasing the strike price of one or more options in the remaining portfolio reduces the executive's exposure and thus increases the moneyness at which the first option is exercised, whereas increasing the time to maturity of one or more options in the remaining portfolio increases the executive's exposure (since the unhedgeable risk is borne for longer), so the moneyness at which the first option is exercised decreases.

Lower exercise thresholds generally lead to a reduction in the cost of an option to the shareholders. ${ }^{2}$ Even for a single option, the lower optimal exercise threshold reduces the ESO cost to the shareholders relative to the Black-Scholes value of a similar traded option. An executive with multiple options will exercise some of these at even lower moneyness thresholds, increasing the proportional discount relative to Black-Scholes still further for these options and so also increasing the proportional discount for the portfolio as a whole. For example (see Tables 1 and 2) combining two at-the-money options with different times to maturity ( 5 and 10 years) can decrease the cost of the 5 -year option from $53 \%$ to $30 \%$ of the Black-Scholes value, a proportional discount of $44 \%$ relative to its cost as a stand-alone option. The portfolio cost is over $20 \%$ lower than the sum of the costs of individual options, and only $40 \%$ of the Black-Scholes world value.

Importantly, the shareholder cost of an option depends on the composition of the remainder of the executive's portfolio. The primary reason is because the moneyness of the early exercise threshold, and hence the shareholder cost depends critically on the option's location in the optimal exercise order. Generally, the later the option is in the exercise order, the higher its moneyness threshold and the higher its $\operatorname{cost}^{3}$ (lower its proportional discount). However,

\footnotetext{
${ }^{2}$ Shareholders are assumed to be well-diversified with no restrictions on trading and thus value ESOs in a perfect market (Black-Scholes world) taking into account the executive's exercise strategy. The lower the exercise threshold, the larger the difference from the Black-Scholes world threshold and the greater the reduction in cost.

${ }^{3}$ If the exercise order changes during the option's life, the longer its position is later in the exercise order, the
} 
the exercise order depends on the strike prices and times to maturity of the options in the portfolio. All else equal, the higher the strike price and the longer the time to maturity, the later in the exercise order an option is. So if the strike prices and times-to-maturity are comonotonic, options are exercised in order of increasing strike price, so options with the lowest strike price (and shortest time to maturity) have the largest proportional cost discounts for shareholders relative to Black-Scholes. However, if the strike prices and times to maturity are not co-monotonic, e.g. a longer-dated option has a lower strike price, the optimal exercise order needs to be calculated as part of the solution. We find options are generally exercised in order of increasing strike price, but options which are close to maturity may be exercised earlier than their strike price would indicate.

These interaction effects cause two new features of ESO costs. Firstly, the shareholder cost of a particular ESO with fixed strike price and time to maturity varies non-monotonically with the strike prices and times to maturity of the other options in the executive's portfolio. Secondly, holding the remainder of an executive's portfolio constant, the shareholder cost of a particular ESO may no longer be monotonically decreasing in its own strike price; it can have local minima. Both findings are in stark contrast to the standard results for exchange-traded options valued under absence of arbitrage, where an option's value is independent of the portfolio in which it is held.

Firms are concerned about the overall cost of options granted to executives, but specifically the cost of the current option grant. A key implication of the nonlinearity of the shareholder cost of ESOs is that this should be measured as the marginal or incremental cost of the grant. In a Black-Scholes world, this would equal its stand-alone value; however with portfolio effects this is no longer true: the incremental cost is always lower than the stand-alone cost, and varies with the executive's existing exposure to unhedgeable firm-specific risk. So firms should recognize that the cost of a given option grant (and indeed the value of that grant to the executive) will vary depending on the executive's existing portfolio of options on the grant date.

Overall then, portfolio effects complicate matters, but they have important implications for exercise thresholds and costs and should not be overlooked. Consider, for example, two executives at the same firm, $\mathcal{A}$ and $\mathcal{B}$, who have the same level of risk-aversion and ESO holdings except that $\mathcal{A}$ holds one additional option grant (she has been employed longer at the firm). Overall the cost of $\mathcal{A}$ 's ESO portfolio is greater than the cost of $\mathcal{B}$ 's, but the proportional discount higher its cost. 
relative to stand-alone values will be greater for $\mathcal{A}$, and the incremental cost to shareholders of $\mathcal{A}$ 's additional option is likely to be substantially lower than the cost of that option as a stand-alone. Furthermore, if it is not optimal for $\mathcal{A}$ to exercise his additional option first, then $\mathcal{A}$ will exercise some, if not all, of the options he has in common with $\mathcal{B}$ at a lower threshold, and hence earlier than $\mathcal{B}$, even though they have the same level of risk aversion. Examining exercise strategies without taking account of portfolio effects might thus erroneously suggest that $\mathcal{A}$ had higher risk aversion than $\mathcal{B}$. Moreover, firms should recognize that the incremental cost to the shareholders of identical unexpected new option grants to the two executives will be lower for $\mathcal{A}$ than $\mathcal{B}$.

Our paper builds on a large number of studies that consider the valuation of ESOs using utility-based techniques. ${ }^{4}$ Lambert, Larcker \& Verrecchia (1991), Huddart (1994), Carpenter (1998), Hall \& Murphy $(2000,2002)$ and Cai \& Vijh (2005) use a certainty equivalent framework to value ESOs assuming the executive's non-option wealth is invested in exogenously specified, non-optimized proportions in stock and risk-free bonds. Henderson (2005), Leung \& Sircar (2009) and Carpenter, Stanton \& Wallace (2010) value a single grant of identical ESO's, allowing optimal investment of the executive's outside wealth in risk-free bonds and a market asset. ${ }^{5}$ Whalley (2008) allows in addition restricted stock holdings and retention of shares on exercise.

Of importance for our paper is the fact that the literature typically considers a single option grant exercised as a block, and does not take into account the portfolio effects we describe. There are several papers however who consider intertemporal or partial exercise of multiple identical options; cf. Jain and Subramanian (2004), Grasselli and Henderson (2009), Leung and Sircar (2009) and Rogers and Scheinkman (2007). These papers identify that the tradeoff between optionality and unhedgeable risk exposure leads to options being exercised gradually over time, however there is no role for different strikes and maturities in their settings.

Whilst much of the literature assumes CARA utility, most notably Carpenter, Stanton \& Wallace (2010) employ constant relative risk aversion (CRRA) to study wealth effects on (single) option exercise and shareholder costs. ${ }^{6}$ They find that the exercise threshold and shareholder

\footnotetext{
${ }^{4}$ Kahl, Liu \& Longstaff (2003) consider the valuation of restricted stock in a similar framework.

${ }^{5}$ See also Detemple \& Sundaresan (1999) and Ingersoll (2006).

${ }^{6}$ Other models to employ CRRA utility include Huddart (1994), Kulatilaka \& Marcus (1994), Hall and Murphy (2002) and Rogers \& Scheinkman (2007) but none of these allow for optimal dynamic partial hedging of nonoption wealth in the market asset. Ingersoll (2006) does allow for dynamic hedging but treats a marginal option position.
} 
cost differ most from their Black Scholes counterparts when wealth is small. Our study of the effect of other options in the portfolio highlights that the greater the number of options, the larger the difference in threshold and the larger the cost discount relative to Black Scholes. One advantage of our model is that option portfolios tend to be observable whereas executive's non-option wealth is typically difficult to identify.

Empirical studies have found support for a number of economic and behavioral factors influencing executives' early exercise decisions in practice (Huddart \& Lang (1996), Heath, Huddart \& Lang (1999), Carpenter, Stanton \& Wallace (2012), Armstrong, Jagolinzer \& Larcker (2007), Klein \& Maug (2010) and Srivastava (2011)). Much of this empirical evidence is consistent with utility models which explains their frequent useage in the literature (eg. early exercise even in the absence of dividends, options exercised earlier at higher volatility firms). Whilst Carpenter, Stanton \& Wallace (2012) recognize that there should be inter-relations between the exercises of different grants to the same executive, thus far only Klein \& Maug (2010) and Armstrong et al (2007) have tested for aspects of portfolio effects explicitly. ${ }^{7}$ In short, there is strong evidence of the explanatory importance of portfolio effects - Klein and Maug (2010) remark "The explanatory power of the option portfolio effects is very large. These effects have been neglected in previous studies, but they are empirically of first-order importance" (p24). As we will explain, our model is able to generate these empirical findings. In contrast, some of the empirical findings of Klein and Maug (2010) and Armstrong et al (2007) cannot be explained by a Black Scholes or single-grant utility based model.

Klein \& Maug (2010) find that the exercise rate increases in the week after an executive has received a grant of new options. ${ }^{8}$ We show that the moneyness of the exercise threshold for whichever option is first-to-be-exercised decreases when another option is unexpectedly added to a portfolio. If some of the executive's existing options are in-the-money, ${ }^{9}$ these option's thresholds will jump down, potentially from above to below the current stock price, causing an increase in their exercise rate. In contrast, under a Black Scholes model, a new grant does not alter the thresholds for existing options, and thus we would not expect a change in the

\footnotetext{
${ }^{7}$ Specifically, Armstrong et al (2007) and Klein and Maug (2010) have used a hazard rate model to estimate exercise rates. Carpenter et al (2012) use a GMM-based model to estimate an exercise function. Earlier studies calibrated exercise thresholds to specific option valuation models (Carpenter (1998), Bettis et al (2005))

${ }^{8}$ Ofek and Yermack (2000) and Srivastava (2011) find related evidence.

${ }^{9}$ These will be exercised before the new option if it is granted at-the-money with the standard ten years to maturity, since they are then co-monotonic.
} 
exercise rate. Similarly, if we were modeling only a single option grant in a utility model, as in the extant literature, we could not make inferences about the increased likelihood of exercise of other options.

The remainder of the paper is organized as follows. Section 2 presents the model. Sections 3 and 4 investigate the implications for the exercise thresholds and costs. Robustness issues are discussed in Section 5 and Section 6 concludes.

\section{Model}

Consider a risk averse executive who is granted $n$ (American-style) finite-lived non-transferable call options on the stock of her company. Her investment opportunities consist of a riskless bond with constant riskless rate $r$ and the market portfolio with price $M_{t}$. The executive is restricted from trading the underlying stock of her company, with price $S_{t} \cdot{ }^{10}$

The set of $n$ options have maturities $T_{W} \leq T_{X}, \ldots, T_{Y} \leq T_{Z}$ and corresponding strikes $K_{W}, K_{X}, \ldots, K_{Y}, K_{Z}$ and we refer to them as option $W, X, \ldots, Y, Z$. The notation $X\left(K_{X}, T_{X}\right)$ denotes option $X$ with strike $K_{X}$ and maturity $T_{X}$. In practice, executive stock options typically have ten year maturities at grant date, but on a given date, the executive may hold a portfolio of options from several grants each with varying time to maturity remaining and different strikes.

The prices follow

$$
\begin{aligned}
\frac{d S}{S} & =(\nu-q) d t+\eta d B \\
\frac{d M}{M} & =\mu d t+\sigma d Z
\end{aligned}
$$

where standard Brownian motions $B$ and $Z$ are defined on a probability space $\left(\Omega, \mathcal{F}, \mathcal{F}_{u}, \mathbb{P}\right)$ where $\mathcal{F}_{u}$ is the augmented $\sigma$-algebra generated by $\left\{B_{u}, Z_{u} ; 0 \leq u \leq t\right\}$ and their instantaneous correlation is $\rho \in[-1,1]$. The volatility of stock returns $\eta$, expected return on the stock $\nu$, proportional dividend yield $q>0$, and expected return $\mu$ and volatility of the market returns

\footnotetext{
${ }^{10}$ Insiders cannot short sell stock as they are prohibited by Section 16-c of the Securities and Exchange Act. For this reason, we use the term executive for the option holder. Our model could also apply to lower-ranked employees - whilst they are not subject to Section 16-c short sale restrictions, costs of short selling may limit their trading. Indeed, evidence of early exercise across all ranks of employees suggests all employees are constrained as to the hedging they can carry out (Bettis et al (2005), Carpenter, Stanton and Wallace (2012)). Bettis et al (2011) provide evidence of the use of derivatives by insiders and find evidence that they are primarily used for information based (insider information) reasons and no evidence that hedging via derivatives is used as diversification against changes in firm risk. Such deals must be reported to the SEC and generally only involve high-ranking executives.
} 
$\sigma$ are all constants. The mean stock return $\nu$ is equal to the CAPM return for the stock, given its correlation with the market, $\nu=r+\beta(\mu-r) ; \beta=\rho \eta / \sigma$. In common with the utility-based ESO literature, we abstract from leverage and dilution considerations, which should not affect our main results. ${ }^{11}$

The restricted executive faces some unhedgeable risk through her option position since $\rho \in$ $(-1,1)$. Allowing the executive to trade in the market asset enables her to partially hedge the risk she faces from her option portfolio. She holds a cash amount $\theta_{s}$ in $M$ at time $s$ and invests the remainder of her wealth at the riskless rate $r$. The executive's trading or outside wealth $W_{u} ; u \geq t$ follows

$$
d W_{u}=\left(r W_{u}+\theta_{u}(\mu-r)\right) d u+\theta_{u} \sigma d Z_{u} ; W_{t}=w
$$

given initial wealth $w$. The remaining unhedgeable risk that cannot be hedged away is represented by $\eta_{e}^{2}=\left(1-\rho^{2}\right) \eta^{2}$ and thus is greater, the lower the (absolute value of) correlation between the stock price and the market.

An executive with an option portfolio as we have described will maximize her expected utility of terminal wealth at $\tilde{T}$ (where we take $\tilde{T} \geq T_{Z}$ ) over the choice of exercise times $\tau^{W}, \tau^{X}, \ldots, \tau^{Y}, \tau^{Z}$ of the options and the choice of outside investment in the market $\theta_{u}$ (satisfying integrability condition $\left.\mathbb{E} \int_{t}^{\tilde{T}} \theta_{u}^{2} d u<\infty\right)$ and bond. We assume the executive has constant absolute risk averse (CARA) utility denoted by $U(x)=-e^{-\gamma x} ; \gamma>0 .{ }^{12}$ Each time an exercise occurs, the cash proceeds ${ }^{13}$ are added to the executive's outside wealth position or trading portfolio (and will continue to be optimally invested in the market and bond until $\tilde{T}$ ). Denote the ordered exercise times by $\tau^{(n)} \leq \ldots \leq \tau^{(1)}$ where $\tau^{(i)}$ is the exercise time at which there are $i$ options remaining in the portfolio. ${ }^{14}$

\footnotetext{
${ }^{11}$ Denis and Rendleman (2008) consider multiple warrant issues and potential dilution effect of each issuance on the remaining warrants. See also the classic works of Constantinides (1984) and Spatt and Sterbenz (1988) on sequential exercise. Their focus is the impact of dilution on costs, whereas our paper explores the interdependencies of different American style options arising from the impact of non-hedgeable risks and the effect of these dependencies on shareholder cost.

${ }^{12}$ Since we treat portfolio effects, we do not simultaneously treat CRRA utility which would further complicate the model. Specifically we would require an additional wealth variable which would increase the dimension of the free boundary problem. We refer to Carpenter et al (2010) for a detailed analysis in a single-exercise model.

${ }^{13}$ Executives tend to sell shares immediately upon exercise (Ofek and Yermack (2000) and Huddart and Lang (1996))

${ }^{14}$ Hence $\tau^{(n)}=\tau^{W} \wedge \tau^{X} \wedge \ldots \wedge \tau^{Y} \wedge \tau^{Z}$ is the earliest time at which an option is exercised, and $\tau^{(1)}=$ $\tau^{W} \vee \tau^{X} \vee \ldots \vee \tau^{Y} \vee \tau^{Z}$ the last time an exercise occurs.
} 
Denote by $\Pi$ the portfolio of unexercised options and by $|\Pi|$ the size of this portfolio or the number of remaining options. For example, if options $X$ and $Z$ remain unexercised then $\Pi=\{X, Z\}$ and $|\Pi|=2$. We also define the shortest maturity left in the portfolio by $T_{\min }=$ $\min \left\{T_{\pi}: \pi \in \Pi\right\}$. The value to the executive $V^{\Pi}\left(u, W_{u}, S_{u}\right)$ with remaining options $\Pi$, current wealth $W_{u}$ and current stock price $S_{u}$ solves the variational inequalities:

$$
\begin{gathered}
V^{\Pi}\left(u, W_{u}, S_{u}\right) \geq \max _{\pi \in \Pi}\left\{V^{\Pi \backslash\{\pi\}}\left(u, W_{u}+\left(S_{u}-K_{\pi}\right)^{+}, S_{u}\right)\right\} \\
\frac{\partial V^{\Pi}}{\partial t}+\sup _{\theta}\left\{\mathcal{L} V^{\Pi}\right\} \leq 0
\end{gathered}
$$

where the differential operator $\mathcal{L}$ is defined by

$$
\mathcal{L}=\frac{\eta^{2} s^{2}}{2} \frac{\partial^{2}}{\partial s^{2}}+(\nu-q) s \frac{\partial}{\partial s}+\rho \theta \sigma \eta s \frac{\partial^{2}}{\partial w \partial s}+\frac{\theta^{2} \sigma^{2}}{2} \frac{\partial^{2}}{\partial w^{2}}+[\theta(\mu-r)+r w] \frac{\partial}{\partial w} .
$$

If the stock is worthless then options are out-of-the-money and the executive optimally invests her current wealth into the market and riskless bond until the terminal date $\tilde{T}$, giving boundary condition $V^{\Pi}\left(u, W_{u}, 0\right)=\mathcal{M}\left(u, W_{u}, \tilde{T}\right)$ where

$$
\mathcal{M}(t, w, \tilde{T})=\sup _{\left\{\theta_{s}\right\}_{t \leq s \leq \tilde{T}}} \mathbb{E} U\left(W_{\tilde{T}} \mid W_{t}=w\right)=-e^{-\gamma w e^{r(\tilde{T}-t)}} e^{-\frac{(\mu-r)^{2}}{\sigma^{2}}(\tilde{T}-t)}
$$

which is simply the indirect utility from the standard Merton (1971) optimal portfolio choice problem. A second set of boundary conditions is obtained at each option maturity,

$$
\forall\{\pi\} \in \Pi ; \quad V^{\Pi}\left(T_{\pi}, W_{T_{\pi}}, S_{T_{\pi}}\right)=V^{\Pi \backslash\{\pi\}}\left(T_{\pi}, W_{T_{\pi}}+\left(S_{T_{\pi}}-K_{\pi}\right)^{+}, S_{T_{\pi}}\right) .
$$

The optimal exercise times $\tau^{(n)} \leq \ldots \leq \tau^{(1)}$ are characterized by ${ }^{15}$

$$
\tau^{(|\Pi|)}=\inf \left\{t \leq u \leq T_{\min }: V^{\Pi}\left(u, W_{u}, S_{u}\right)=\max _{\pi \in \Pi}\left\{V^{\Pi \backslash\{\pi\}}\left(u, W_{u}+\left(S_{u}-K_{\pi}\right)^{+}, S_{u}\right)\right\}\right\}
$$

This says an option from an existing portfolio $\Pi$ is exercised when the value from continuing to hold it is sufficiently low that it equals the value from exercising a particular option $\pi$ (and continuing with the portfolio without this option where the payoff $S_{u}-K_{\pi}$ is optimally invested in the bond and market). The option that is exercised from the portfolio is the one that gives the maximum continuation value for the remaining portfolio, including the reinvestment of the

\footnotetext{
${ }^{15}$ When only one option remains, $|\Pi|=1$ and the optimal exercise time is given by

$$
\tau^{(1)}=\inf \left\{t \leq u \leq T_{\pi}: V^{\Pi}\left(u, W_{u}, S_{u}\right)=V^{\emptyset}\left(u, W_{u}+\left(S_{u}-K_{\pi}\right)^{+}, S_{u}\right)\right\}
$$

where $V^{\emptyset}\left(u, W_{u}, S_{u}\right)=\mathcal{M}\left(u, W_{u}, \tilde{T}\right)$.
} 
option proceeds. In the Appendix we give details of the separation of variables, transformations and numerical methods to solve the above free boundary problem.

The solution to the problem involves finding the exercise threshold $S^{(|\Pi|)}(t)$ for the next option to be exercised when the portfolio contains the remaining options $\Pi$. These are given in (17) (see Appendix). As with standard American options, we can then describe the optimal exercise time for the next option when $|\Pi|$ remain as the first time the stock price reaches this exercise threshold,

$$
\tau^{(|\Pi|)}=\inf \left\{t \leq u \leq T_{\min }: S_{u}=S^{(|\Pi|)}(u)\right\}
$$

Once we have solved for each threshold $S^{(|\Pi|)}(t)$, we can infer the optimal exercise boundary for each option $\pi \in \Pi$ calculated as part of the portfolio, denoted $S^{\pi}(t) ; t \leq T_{\pi}$. The corresponding optimal exercise time for each option $\pi \in \Pi$ is

$$
\tau^{\pi}(t)=\inf \left\{t \leq u \leq T_{\pi}: S_{u}=S^{\pi}(u)\right\}
$$

For comparison, we also calculate the exercise thresholds $S_{\mathcal{S}}^{\pi}(t)$ of each option as a standalone, ie. if the portfolio consisted of a single option. These are computed via (17) with $|\Pi|=1$.

Finally, following Henderson (2005), Carpenter et al (2010), Leung and Sircar (2009) amongst others, we define the subjective value $p^{\Pi}(t, s)$ of the portfolio of remaining options $\Pi$ to the executive as the amount of cash which invested optimally would give the same expected utility as the options ${ }^{16}$

$$
V^{\Pi}(u, w, s)=\mathcal{M}\left(u, w+p^{\Pi}(u, s), \tilde{T}\right)
$$

Again, the subjective value of a particular option as a stand-alone is computed with $|\Pi|=1$.

\section{Exercise thresholds}

We first focus on the executive's optimal exercise thresholds since option exercises are observable in the market, and are inputs into both the executive's valuation and the shareholder's costs of the option portfolio. Exercise decisions are of independent interest as they may give insights into executive preferences and lead to benchmarks useful in broader corporate finance research.

Since our focus in this paper is on impact of different option strikes and maturities we take a portfolio with $n=n_{1}+n_{2}$ options where $n_{1}=n_{2}=1$. This will be our base portfolio for

\footnotetext{
${ }^{16}$ In the Appendix, we characterize the free boundary problem that $p^{\Pi}(t, s)$ must solve, see (19)-(21).
} 
much of the remainder of the paper. Later in Section 5 we will extend to consider portfolios with further options.

It is well known that the exercise threshold for a single executive stock option lies below the equivalent threshold in a Black-Scholes setting, because of the effect of unhedgeable risk on the executive's option value. Whilst she continues to hold the option, the executive bears additional risk due to her exposure to the firm's stock price, which she is unable perfectly to hedge. On exercise however, the executive sells the shares she receives and thus eliminates her exposure to this unhedgeable risk. The unhedgeable risk exposure reduces the value of continuing to hold the option to the executive and so decreases the threshold at which she optimally exercises the option.

\subsection{A Single Grant}

Figure 1 shows how the exercise thresholds evolves over time for a portfolio of two identical options, both with strike $K_{Y}=10$ and maturity $T_{Y}=10 .{ }^{17} 18$ The dashed line is the executive's optimal threshold taking account of unhedgeable risk for a single option as a stand-alone. It is significantly lower than the highest solid line, which represents the Black-Scholes world threshold, for all times to maturity, showing how unhedgeable risk decreases the level of the threshold throughout the option's life. Of course the Black-Scholes world threshold remains the same regardless of whether the executive is exercising one or many identical options, since all risk is hedgeable and there is no need to unwind risk gradually over time.

When determining whether to exercise the option, the executive trades off the benefits of continuing to hold the option (interest on the deferred payment of the strike price), with the costs, which include not only the foregone dividend yield, as in the Black-Scholes world, but also the cost of the unhedgeable risk exposure. ${ }^{19}$ The additional cost of ongoing unhedgeable risk exposure reduces the threshold from the Black-Scholes threshold, by more, the greater the executive's risk aversion and the greater the risk to which she is exposed..$^{20}$ Furthermore, the effects of unhedgeable risk increase with the length of time the risk is borne. So whilst the convexity

\footnotetext{
${ }^{17}$ We take $\rho=0$, so all risk is unhedgeable.

${ }^{18}$ In practice, ESO's commonly have maturities of ten years.

${ }^{19}$ We compare the partial differential equation for the option value (see Appendix, (19)) with the Black Scholes pde given in (24). The additional term representing the effects of unhedgeable risk in (19) is like a non-constant increase in the dividend yield which scales with the executive's risk aversion, $\gamma$, the unhedgeable variance of the stock price, $\eta^{2}\left(1-\rho^{2}\right)$, and her exposure to this, measured by the option delta.

${ }^{20}$ For more details see Sun (2011).
} 
of the option payoff still means that the option value to the executive and hence the optimal exercise threshold increases rapidly as the time to maturity increases from zero, the counteracting reduction in option value due to unhedgeable risk means the threshold stops increasing at a lower time to maturity and can even decrease as the time to maturity increases. (See also Carpenter et al (2010)). Again, the difference in the slopes of the exercise threshold $v s$ time for long times to maturity increases with the executive's risk aversion, the stock's unhedgeable risk and the executive's exposure to that risk, and also with the riskfree rate.

The dashed line in Figure 1 represents the optimal exercise threshold for this option not only as a stand-alone option but also when it is part of any portfolio in which it will optimally be the last option to be exercised. Once all the executive's other stock options have been exercised, the executive takes into account only the unhedgeable risk associated with the remaining, single option, which will be eliminated on exercise, and exercises at the same threshold as for a standalone option. (We characterize the portfolio combinations for which this is the case in more detail soon.) More generally, when deciding when to exercise a particular option, the executive takes into account the reduction in her exposure to unhedgeable risk which occurs upon exercise and sale of the resulting shares. Since the effects of unhedgeable risk are related to the variance of the unhedgeable risk exposure, they increase nonlinearly with the size of the option portfolio. So the reduction in unhedgeable risk exposure on exercise of a given option is generally greater, the larger the overall option portfolio. For example, exercising the first option in a portfolio of two identical options reduces the executive's exposure to unhedgeable risk by considerably more than exercising the second (last) option. The lowest dot-dashed line in Figure 1 shows the optimal exercise threshold for the first-to-be-exercised option in such a portfolio. As we see in Figure 1, the executive prefers to unwind risk gradually over time by exercising options at different thresholds, in agreement with Leung and Sircar (2009), Carpenter et al (2010) and Grasselli and Henderson (2009). The greater impact of unhedgeable risk can be seen both in the reduction in the threshold instantaneously before maturity (and hence the overall level of the threshold) and in the larger cumulative effects for long times to maturity (the slope far from maturity).

\subsection{Which option is exercised first?}

We have seen that under utility based valuation, the exercise order has a significant impact on the level of the exercise threshold. But what determines the exercise order? In a Black-Scholes world, 
holding all else constant, options with lower strike price (or equivalently, greater moneyness) and shorter times to maturity are exercised earlier. If these two effects work in the same direction, so options in a portfolio have co-monotonic strike prices and times to maturity, then options are exercised in order of increasing strike (Chapter 4 of Cox and Rubinstein (1985)). Henderson et al (2012) have shown that this co-monotonicity result continues to hold under utility-based pricing in incomplete markets ${ }^{21}$ i.e. for an executive with a portfolio of two options, $Y$ and $Z$, if $K_{Y} \leq K_{Z}$ and $T_{Y} \leq T_{Z}$ then it is always optimal for the executive to exercise $Y$ before $Z$. Given ESO's are most often granted at-the-money, if there is a bull market, an executive will typically have a portfolio of options where the more recent options have a higher strike price and a longer time to expiry. In this situation we show it is always optimal to exercise the "older" options first since they also have the lower strikes. However, if $T_{Y} \leq T_{Z}$ but $K_{Z}\left(<K_{Y}\right)$ is sufficiently small, then it can be optimal never to exercise $Y$ before $Z$. More generally, the optimal exercise order can switch during the life of the shorter dated option. These situations will arise in a bear market when portfolios will tend to have more recent options with a lower strike price but longer time to expiry.

The left panel of Figure 2 shows the exercise thresholds for a portfolio of two options, $Y$ and $Z$, which are co-monotonic with $K_{Y}=10, T_{Y}=5, K_{Z}=11, T_{Z}=10$. The thresholds for option $Y$ are solid lines, whereas those for option $Z$ are dashed. The top pair of lines represent the Black-Scholes thresholds for options $Y$ and $Z$. The lower pair of lines represent the thresholds under utility-based valuation. Red lines represent thresholds at which the first option and blue lines thresholds at which the second or last option in the portfolio is exercised. Since $Z$ is always exercised after $Y$, the dashed (blue) threshold at which option $Z$ is exercised is equivalent to a stand-alone threshold for $Z$. However, the solid (red) threshold for $Y$ reflects the presence of $Z$ and is not equivalent to $Y$ 's stand-alone exercise threshold. In contrast, the Black Scholes thresholds for each option do not change in the presence of other options in the portfolio and thus are the same as stand-alone thresholds for each individual option.

The right panel of Figure 2 lowers the strike of $Z$ to $K_{Z}=4$, so that the option strikes and maturities are not co-monotonic. Now we see that under our model, it is never optimal to exercise $Y$ first. The dashed threshold for option $Z$ is always lower than the solid (blue) threshold for option $Y$. As in a Black-Scholes setting, the effects of differences in times to maturity are

\footnotetext{
${ }^{21}$ In fact American call options with co-monotonic strikes and maturities will be exercised in order of increasing strike under very minimal assumptions on prices and preferences, see Henderson et al (2012) for details.
} 
more important when the options are closer to maturity, ${ }^{22}$ whereas for long times to maturity, differences in time to maturity become relatively less important, so differences in strike prices determine the exercise order. In this case, $K_{Z}$ is sufficiently low that the strikes determine the exercise order for the whole life of the shorter dated option, so $Z$ is always exercised first. ${ }^{23}$

For some strike/maturity combinations, the tradeoff between the strike/moneyness and the time-to-maturity changes such that the exercise order switches from initially exercising the option with a longer time to maturity first, say $Z$ with lower strike, to exercising the shorter dated option (with the higher strike price), $Y$ say, first. We denote the switchover time by $T^{*} \in\left[0, T_{Y}\right] \cdot{ }^{24}$ If $K_{Z}$ is sufficiently small, as above, that it is never optimal to exercise $Y$ before $Z$, then $T^{*}=T_{Y}$. Alternatively, if $K_{Y} \leq K_{Z}$ so we are in the co-monotonic case, then $T^{*}=0$.

Figure 3 shows the exercise thresholds for a portfolio of two options, $Y$ and $Z$, which are not co-monotonic and where the exercise order switches during the life of the option. Option $Y$ has a higher strike price but a shorter time to maturity than option $Z: K_{Y}=10>8=K_{Z}$, $T_{Y}=5<10=T_{Z}$. As before, the top pair of thresholds are the equivalent Black Scholes thresholds which cross at $T_{B S}^{*}=1.09$. The optimal Black Scholes strategy is to exercise option $Z$ first if $0 \leq t \leq 1.09$ and option $Y$ first if $1.09 \leq t \leq 5=T_{Y}$. The optimal exercise order also switches under utility-based valuation (the lower set of thresholds) from exercising $Z$ first to exercising $Y$ first, though at a later time, $T^{*}=4.25$. However, in contrast to the Black-Scholes setting, where the switch in the exercise order has no impact on the location of either exercise threshold, the difference in the level of each option's exercise threshold depends on whether it is exercised first or last (more generally, later) and results in a discontinuous jump in the optimal exercise threshold for each individual option, $S^{Y}$ and $S^{Z}$, over the switching time, $T^{*} . S^{Z}$ is lower before $T^{*}$, when $Z$ is exercised first, i.e. before option $Y$, than afterwards, and so 'jumps up' over the switching time as $Z$ becomes the last to be exercised option. Correspondingly, $Y$ 's

\footnotetext{
${ }^{22}$ Exercise thresholds change rapidly with time to maturity when the time to maturity is short.

${ }^{23}$ In the Black Scholes world, we see the thresholds for $Y$ and $Z$ intersect close to the maturity of $Y$. $Z$ is exercised first before this intersection, then immediately prior to $Y$ 's maturity it is optimal to exercise $Y$ first. Again, however, the Black Scholes thresholds do not change in the presence of other options, and thus the thresholds are the same as they would be for each option as a stand-alone.

${ }^{24}$ For a portfolio consisting of options $Y$ and $Z$ with $T_{Y} \leq T_{Z}$, the switchover time $T^{*}$ is defined via:

$$
T^{*}=\inf \left\{u \leq T_{Y}: e^{-\gamma\left(1-\rho^{2}\right)\left(S_{u}-K_{Y}\right)^{+} e^{r(\tilde{T}-u)}} H^{Z}\left(u, S_{u}\right) \leq e^{-\gamma\left(1-\rho^{2}\right)\left(S_{u}-K_{Z}\right)^{+} e^{r(\tilde{T}-u)}} H^{Y}\left(u, S_{u}\right)\right\}
$$
}


threshold, $S^{Y}$ 'jumps down' at $T^{*}$ as $Y$ becomes first to be exercised. ${ }^{25}$

Figure 3 also shows a dotted (green) line, which represents the threshold for option $Y$ if option $Z$ is exercised before $T^{*}$. Since the portfolio consists of only two options, this is the same as the stand-alone threshold for $Y$. In order to explain the thresholds in more detail, we consider various scenarios for stock prices, pictured in Figure 4. In the left-hand panel, both Path A and Path B cross the lowest exercise threshold (for option $Z$ ) before $T^{*}$, so option $Z$ is exercised first. The executive's portfolio then consists just of option $Y$, which has exercise threshold as for a stand-alone option, given by the combination of the solid (blue) line for $t<T^{*}=4.25$ and the (green) dotted line for $T^{*} \leq t<T_{Y}$. Stock path A crosses this line so option $Y$ is exercised, whereas stock path B remains below it and option $Y$ expires unexercised. In contrast, the stock paths in the right-hand panel do not cross the lowest exercise threshold before $T^{*}$. Between $T^{*}$ and $T_{Y}$ it is optimal to exercise $Y$ first if the stock price crosses the lower solid (red) line. Stock path A does, so option $Y$ is exercised; stock price B does not, so option $Y$ expires unexercised. Once option $Y$ has either expired or been exercised the executive's portfolio consists solely of option $Z$ and is exercised only if (as is the case for both stock price paths) the stock price crosses its stand-alone exercise threshold given by the dashed (blue) line before its maturity $T_{Z}$. Thus which exercise threshold is relevant for option $Y$ between $T^{*}$ and $T_{Y}$ depends on the stock price behavior before $T^{*}$ (it is the dotted (green) line if $Z$ has been exercised before $T^{*}$, or the lower, solid (red) line if not).

The exercise threshold for either one of the two options in this case exhibits significantly different characteristics from those under Black-Scholes. Not only is the threshold no longer monotonically increasing with time to maturity but strikingly, it exhibits a discontinuity whenever the exercise order changes. ${ }^{26}$

To summarize, for a portfolio of two options there are three possible forms of exercise strategy. First, if the strike price of the longer-maturity option is sufficiently high relative to that of the

\footnotetext{
${ }^{25}$ More generally, at any switching time, any option whose order switches will have a discontinuity in its exercise threshold. The threshold will be significantly higher after the switching time if the option becomes later in the exercise order, and will decrease significantly if it becomes earlier in the exercise order

${ }^{26}$ For the relatively longer dated option, $Z$, the exercise threshold whilst it is the first-to-be-exercised option increases as time to maturity decreases and, particularly close to the switchover time, at an increasing rate, so the exercise threshold is convex in time to maturity. After the discontinuity in the threshold when the exercise order switches, once the option has become the last-to-be-exercised, the threshold may still increase for long enough times to maturity. The relatively shorter dated option, $Y$, can start as the last-to-be-exercised with a threshold which may increase or decrease with time to maturity.
} 
shorter-maturity option (e.g. the strike prices and times to maturity are co-monotonic), then it is always optimal to exercise in order of increasing time to maturity, i.e. exercise the shortermaturity option before the longer-maturity option as long as they are both alive, i.e. $\left.t<T_{\min }\right)$. The longer-maturity option is thus exercised first only after the expiry of the shorter-maturity option, i.e. $T^{*}=0$. On the other hand, if the strike price of the longer-maturity option is sufficiently low so that for example it is optimal to exercise it first even at the expiry of the shorter-maturity option, then the strategy is to exercise in order of increasing strike price, i.e. exercise the longer-maturity option before the shorter-maturity option at all times, even before the shorter-maturity option expires. So $T^{*}=T_{\min }$. Finally, for intermediate values of the strike price of the longer-maturity option relative to that of the shorter-maturity option, it is initially optimal to exercise in order of increasing strike price, i.e. exercise the longer-maturity option with lower strike price first, but the optimal exercise order switches before expiry of the shorter-maturity option to exercising the longer maturity option last, so $0<T^{*}<T_{\min }$.

The combinations of strike price and maturity for which each strategy holds are illustrated in stylized form in Figure 5. This illustrates the optimal strategy for a portfolio consisting of an option with strike price $K$ and maturity $T$ together with our base-case option $Y$, with $K_{Y}$ and $T_{Y}$ for different combinations of $(K, T)$. The form of the strategy depends on which region, A $\mathrm{F}$, the strike price and maturity of the second option in the portfolio fall into relative to option $Y$. The vertical and horizontal quadrant lines represent the boundaries of the co-monotonic regions. So in the top right quadrant, B, $Y$ is always exercised first and the later maturity option is exercised last, as long as both options are still alive, i.e. $T^{*}=0$. Similarly in the bottom left quadrant $\mathrm{E}$ where $K \leq K_{Y}$ and $T \leq T_{Y}$, whilst both options are still alive, the earlier maturity option is exercised first and option $Y$ is exercised last. The curved solid line represents, for each maturity $T$, the maximum, if $T>T_{Y}$, (minimum if $T<T_{Y}$ ) strike price for which it is always optimal to exercise the option with the lower strike price first (even though this has a longer time to maturity).

In the top left and bottom right quadrants the effects of strike prices and maturities pull in opposite directions. In areas $\mathrm{A}$ and $\mathrm{F}$ the impact of the difference in strike prices dominates, so in area A, option $Y$ is always exercised before the shorter-maturity option due to $Y$ 's lower strike price, and in F, option $Y$ has higher strike price and is always exercised last. In these areas, $T^{*}=\min \left(T_{Y}, T\right)$. In areas $\mathrm{C}$ and $\mathrm{D}$ the strike price difference dominates initially, but there is a switch in the optimal exercise order at $0<T^{*}<T_{\min }$ so for times close to $T_{\min }$ the 
differences in maturity times dominate. ${ }^{27}$

The overall effect is that in the darker shaded regions A and B the optimal strategy whilst both options are still alive is to exercise $Y$ first, whereas in the lighter shaded regions $\mathrm{E}$ and $\mathrm{F}$ where there is a choice, it is optimal to exercise $Y$ last. In regions $\mathrm{C}$ and $\mathrm{D}$, which option it is optimal to exercise first depends on when the stock price first crosses the threshold of whichever is the first-to-be-exercised option. When the exercise order changes at $T^{*}$, there is a jump in the thresholds for each individual option. Only in the co-monotonic regions, B and E, are there no jumps in either threshold.

Within each region, although the form of the exercise strategy remains the same, the threshold for any option except the last-to-be-exercised varies with the composition of the remainder of the executive's portfolio. For example, Figure 6 shows the effect of different strike prices for a longer dated option, $Z$, with $T_{Z}=10$, on the exercise threshold for a 5-year option $Y$ with $K_{Y}=10$ when held together in a portfolio. The top, solid line is the threshold if $Y$ is always exercised last, which equals the stand-alone threshold. This is $Y$ 's threshold if $K_{Z}$ is sufficiently low (any point in region $\mathrm{F}$ in Figure 5) and it is unaffected by changes in $K_{Z}$. As $K_{Z}$ increases, it becomes optimal to exercise $Y$ first when it is sufficiently close to maturity (region D). For example, for $K_{Z}=8.5$, the switchover time for these parameter values is $T^{*}=3.19$, so before then, $Y$ is still exercised last and the threshold coincides with the top, stand-alone threshold; however for $T^{*}<t<T_{Y}$, it is optimal to exercise $Y$ first at a much lower threshold. As $K_{Z}$ increases further to $K_{Z}=9$, there are two effects. Firstly, $Y$ is optimally exercised first over a longer period, so the switchover time, $T^{*}$, decreases (to 1.39). $Y^{\prime}$ 's threshold is the lower, first-to-be-exercised threshold for longer. Secondly, the first-to-be-exercised threshold itself is slightly higher than when $K_{Z}=8.5$. This reflects the lower marginal reduction in unhedgeable risk on $Y$ 's exercise due to $Z$ 's lower moneyness. When $K_{Z}=10$, the options are co-monotonic so it is always optimal to exercise $Z$ first (Region $\mathrm{B}$ with $T^{*}=0$ ). However the effect of $Z$ 's moneyness on $Y$ 's first-to-be-exercised threshold continues to hold. So $Y$ 's first-to-be-exercised threshold for $K_{Z}=10$ is higher than that for $K_{Z}=9$ and that for $K_{Z}=11$ is higher still.

Thus far we have illustrated the forms of the exercise strategy and the resulting exercise thresholds for particular parameter values. The potential forms of the exercise strategy, the

\footnotetext{
${ }^{27}$ Particularly for short times to maturity, the boundary between regions $\mathrm{B} / \mathrm{D}$ and $\mathrm{C} / \mathrm{E}$ i.e. the strike price at which it is just optimal to exercise shorter-maturity option first for all remaining $t<T_{\min }$ may differ from the horizontal lines shown in the figure, so region B may extend below $K_{Y}$ and region E may extend above $K_{Y}$. Horizontal lines represent boundaries for all times.
} 
shape of the regions of relative strike prices and maturity dates, within which each strategy applies, and the general shape of the exercise thresholds all remain unchanged when parameter values are varied. Many of the new features in the shape and (lack of) continuity of the exercise thresholds vs time are due to the effect of the executive's exposure to unhedgeable risk. So changes in parameter values which increase the magnitude of the effect of this exposure to unhedgeable risk increase the effects on the exercise thresholds. If the magnitude of the effect increases, all exercise thresholds decrease because of the increased cost; however, due to diminishing returns to scale the exercise thresholds of the last-to-be-exercised options decrease more than those of the second-to-last (first) to be exercised options, decreasing the magnitude of the jump in the thresholds when the exercise order changes. More importantly, since the effect of unhedgeable risk exposure increases with the length of time the unhedgeable risk must be borne, the exercise thresholds for longer-maturity options decrease more then those of shorter maturity options, both when last- and when first-to-be-exercised. Hence the switchover time, $T^{*}$, increases, so longer-maturity options are exercised first for a longer initial period. In unreported simulations, these effects are found to hold consistently for increases in risk aversion, $\gamma$, and in the risk-free rate, $r$, which increases the impact of the unhedgeable risk exposure over time. Increases in the dividend yield, $q$, have similar effects, since exercise thresholds decrease due to the increased foregone dividend yield, though less markedly since the increase in this effect over time is less pronounced.

\subsection{Implications}

Exercise thresholds are important because they are observable: the exercise of an ESO reveals one point of the empirical exercise threshold of the first option to be exercised from the executive's ESO portfolio. This is usually described in terms of the relative moneyness on exercise or $S^{\pi} / K$. So what are the implications of our model for the relative moneyness on exercise (hereafter moneyness) of ESOs held in portfolios, specifically the first-to-be-exercised moneyness which is observed in practice?

Most implications follow directly from the equivalent results for exercise thresholds themselves. So for example, for any option in a portfolio (as long as it is not exercised last), the moneyness on exercise is lower than the moneyness on exercise for the same option held as a stand-alone. A particular ESO's, say Y's, moneyness on exercise decreases if the executive is granted additional options optimally exercised after $Y$. It also varies with the terms $(K, T)$ of 
other options in the executive's portfolio after it in the optimal exercise order.

Like the threshold itself, the moneyness on exercise of the first-to-be-exercised ESO is generally non-monotonic with respect to time to maturity, first increasing, potentially at an increasing rate, and then decreasing as time gets closer to the maturity of the each option in the portfolio. It jumps when the identity of the first-to-be-exercised option changes during the portfolio's life: generally jumping up on exercise or expiry of the first-to-be-exercised ESO and jumping down if there is additionally a switch in the optimal exercise order whilst the portfolio composition remains unchanged. ${ }^{28}$ For example, the portfolio of $Y$ and $Z$ in Figure 3 with $K_{Y}=10, T_{Y}=5$ and $K_{Z}=8, T_{Z}=10$ has switching time, $T^{*}=4.25 . Z$ is exercised first before $T^{*}, Y$ afterwards. At $T^{*}$ the exercise threshold of the first-to-be-exercised option, at which the executive is indifferent between exercising $Y$ first or $Z$ first, is 12.49 . The relative moneyness on exercise of the first option to be exercised thus drops from $12.49 / K_{Z}=1.56$ for exercising $Z$ just before the switchover to $12.49 / K_{Y}=1.25$ for exercising $Y$ just afterwards.

The non-monotonicity and jumps in the relative moneyness on exercise of the first-to-beexercised ESO in a portfolio makes it difficult to draw empirical predictions about the determinants of observed moneyness on exercise which hold in all circumstances. However, empirical studies of ESO exercise in practice often work with exercise rates or propensity to exercise, and we show how our model can be used to explain empirical exercise patterns around new option grants (Section 3.3.1) and other empirical findings on exercise rates (Section 3.3.2).

\subsubsection{Immediate exercise of existing options: the impact of a new grant}

Several empirical studies have found a relation between the executive's propensity to exercise existing options and the arrival of a new grant of options. Specifically, Ofek \& Yermack (2000) find that executives with larger exposure to firm-specific risk (larger portfolios of stocks and options) were more likely to exercise some existing options after new option grants than executives with lower prior exposure. Similarly, Klein \& Maug (2010) also find that new option grants increase executives' propensity to exercise, and Srivastava (2011) finds that existing options' time to maturity on exercise increases with the size of new option grants.

Under a Black Scholes framework, a new grant does not alter the thresholds of the existing options, and thus cannot alter the likelihood that any existing options will be exercised. In

\footnotetext{
${ }^{28}$ Recall that after the switch, the option exercised first will have a shorter time to maturity and higher strike price.
} 
contrast, the increase in unhedgeable risk brought to the portfolio by the new grant means that it may be optimal to immediately exercise the existing option in our setting. Indeed, as we demonstrate, the decrease in the relative moneyness required to trigger immediate exercise once executives' risk-aversion and portfolio effects are taken into consideration is consistent with these results.

Consider an executive with an existing option $Y$ who receives a new option grant $Z$. If $K_{Z}$ is sufficiently high that it is optimal to exercise $Y$ first as soon as the new option, $Z$, is granted, $Y$ 's threshold once the new option has been added to the portfolio will be much lower than its threshold in the absence of the new option. New options are often granted at-the-money. So if option $Y$ is sufficiently in-the-money, then an unexpected new at-the-money option grant may induce $Y$ 's immediate exercise. The level of moneyness required for exercise once $Y$ is part of the new, larger portfolio will be much lower than that required for $Y$ as a stand-alone option.

For example, consider option $Y$ with $K_{Y}=10, T_{Y}=5$. For parameters given by $\gamma=0.1, r=$ $0.05, q=0.02, \eta=0.4, Y$ is exercised immediately under a Black Scholes framework if the stock exceeds 48.8, or equivalently, $Y$ 's relative moneyness $S / K_{Y}$ exceeds 4.88. The equivalent standalone threshold for $Y$ in moneyness terms is 1.65. (This takes into account only the unhedgeable risk associated with option $Y$ itself.) Now consider receiving another option $Z$ with $T_{Z}=10$. The presence of $Z$ in the portfolio dramatically lowers $Y$ 's moneyness threshold such that $Y$ is exercised immediately if $S / K_{Y}>1.39$. Hence the probability of immediate exercise of existing options at the date of a new option grant is higher once risk-aversion and unhedgeable risk with portfolio effects are taken into account. Factors that increase unhedgeable risk will reduce $Y$ 's moneyness threshold and raise the likelihood that the existing option is exercised immediately at the grant date of the new option.

\subsubsection{Empirical literature on ESO exercise rates}

A number of papers have investigated exercise thresholds and rates for ESOs. For example Carpenter, Stanton \& Wallace (2012) recognize the complexity of stock option portfolios in practice in the methodology they use but do not include specific controls for the composition of the remainder of the portfolio. Only Klein \& Maug (2010) and Armstrong et al (2007) test explicitly for portfolio effects. They find strong evidence of the explanatory importance of portfolio effects. Klein \& Maug (2010) find that most of the options exercised have a time value 
which is lowest or close to lowest of all options in the executive's portfolio ${ }^{29}$ and Armstrong et al (2007) find relationships between the exercise rate of a particular option and the intrinsic values of other options in an executive's portfolio. Both are consistent with the portfolio effects we describe.

Armstrong et al (2007) show that the exercise rate of a particular option, say option $Y$, is decreasing in the overall intrinsic value of the executive's other option grants, if this is positive, and is also decreasing in the absolute value of this intrinsic value if the intrinsic value is negative. Although seemingly inconsistent, both of these results can be explained using our model. To illustrate the main effects, we approximate the remainder of the option portfolio by a holding with common strike price and time to maturity. First, if the intrinsic value of the executive's other options is negative, they are out-of-the-money, and so are most likely to be exercised after $Y$. Increasing the strike price of the executive's other options increases the magnitude of the other options' (negative) intrinsic value. This decreases the unhedgeable risk associated with these other options and therefore also decreases the reduction in the unhedgeable risk on exercising $Y$. This leads to an increase in $Y$ 's optimal exercise threshold or equivalently a decrease in $Y$ 's exercise rate, as in Armstrong et al (2007). In the other case when the intrinsic value of the executive's other options is positive, $Y$ may be exercised before or after the other options. The primary effect is that a decrease in the strike price of the other options, which increases their intrinsic value, can lead to a change in the optimal exercise order, so $Y$ is exercised first before the change in the strike price but is exercised later (last) afterwards. We show that the optimal exercise threshold for an option jumps up when it moves to a later position in the exercise order, due to the sudden decrease in the change in unhedgeable risk arising from the exercise of this particular option. This is equivalent to a decrease in the option's exercise rate, as found by Armstrong et al (2007). ${ }^{30}$

\footnotetext{
${ }^{29}$ They calculate the time value using Black-Scholes world values, so the optimal exercise ordering may sometimes differ from the ordering of the Black-Scholes world time values on exercise. However in many cases (e.g. co-monotonic) the two will coincide, so this is broadly consistent with our model.

${ }^{30}$ Whilst the change in $K$ can increase an option's exercise rate if $Y$ is exercised first both before and after the change in strike price, using a similar argument to the case of negative intrinsic value, the magnitude of this effect is much smaller, since the change in the exercise threshold is incremental rather than a discontinuous jump. The number of portfolios to which it applies is also small, since it is limited by the requirement that the intrinsic value remain positive.
} 


\section{Shareholder Costs}

Shareholders do not face the same restrictions on trading as executives, so they value them in a perfect market setting (Black-Scholes world), but taking account of the executive's optimal exercise threshold (See Carpenter (1998), Carpenter, Stanton \& Wallace (2010) amongst others). The cost of each option, $C^{\pi}(t, s)$, is calculated using the Black-Scholes equation whilst the stock price remains below the optimal exercise threshold, $S^{\pi} .{ }^{31}$ The portfolio cost, $C^{\Pi}(t, s)$, is given as the sum of the individual option costs, $C^{\pi} \cdot{ }^{32}$

\subsection{Portfolio effects: Discount relative to Black-Scholes costs}

Decreases in the executive's optimal exercise thresholds due to the presence of other options in her portfolio automatically decrease the cost of those options to the shareholders. So the cost of any option when held in a portfolio is generally lower than its cost as a stand-alone. The only exception is if the option is optimally exercised last throughout its remaining life (when the cost is unaffected by the presence of other options in the portfolio). As a consequence, the overall shareholder cost of any portfolio of options is lower than the sum of the costs of each option evaluated on a stand-alone basis, since all bar at most one of the options making up the portfolio are exercised earlier and thus have lower individual costs. It is well known that the costs of individual ESOs are lower than their equivalent perfect market (Black Scholes world) values due to the executive's optimal early exercise in the presence of unhedgeable risk (Carpenter, Stanton \& Wallace (2010) or Leung \& Sircar (2009)). Portfolio effects increase the magnitude of this reduction: a greater discount is required from the Black-Scholes world cost to obtain the shareholder costs of ESO portfolios for a given level of executive risk aversion. ${ }^{33}$

For example, consider a five year at-the-money ESO $Y$ on a stock with current stock price $S=10$, dividend yield $q=5 \%$ and (unhedgeable) volatility $\eta=40 \%$, exercisable at any time before its maturity, with risk-free rate $r=10 \%$. The Black-Scholes world value of this grant is 3.48. For a stand-alone ESO grant, taking into account the executive's risk-aversion, $\gamma=0.2$, reduces $Y$ 's cost by $47 \%$ to 1.85 . However, if the executive owning $Y$ also owns a second option, $Z$, which is also vested and has the same strike price but with 10 years to maturity, then the

\footnotetext{
${ }^{31}$ The p.d.e. and boundary conditions for $C^{\pi}(t, s)$ are given in the Appendix.

${ }^{32}$ For example, $C^{Y, Z}$ is the cost of the portfolio of options $Y$ and $Z, C^{Y}$ is cost of $Y$ when considered as part of the portfolio, $C^{Z}$ is the cost of $Z$ as part of the portfolio. The costs can also be calculated for individual options on a stand-alone basis, denoted for e.g. $C_{\mathcal{S}}^{Y}$ by replacing the threshold $S^{Y}$ with the stand-alone threshold $S_{\mathcal{S}}^{Y}$.

${ }^{33}$ Equivalently, a lower level of risk aversion is required to achieve the same discount.
} 
cost of $Y$ decreases further to 1.04, a 70\% reduction relative to the original Black-Scholes world value. The cost of the ESO portfolio, 3.12, (the sum of the portfolio costs), is also less than the sum of the costs of each ESO as stand-alone options, 3.93, again increasing the proportional discount relative to Black-Scholes world values (from $49 \%$ to $60 \%$ ). ${ }^{34}$

These proportional discounts can be significant in magnitude. Table 1 shows how they vary with the executive's risk aversion, $\gamma$, and the firm's idiosyncratic volatility, $\eta_{e}$ and dividend yield, $q$, through $r-q$. Proportional discounts increase, the greater the portfolio effect on the exercise threshold, i.e. the greater the unhedgeable risk $(\eta)$, the greater the cost of that risk to the executive (measured by the risk aversion, $\gamma$ ) and the greater the potential reduction in threshold (the higher the original threshold), so the lower the dividend yield, $q$ (the higher $r-q)$. Overall, portfolio effects increase discounts for the costs of ESOs relative to BlackScholes values, even for low levels of risk aversion (for $\gamma=0.01$, the discount for individual options almost quadruples from $3 \%$ to $11 \%$, and the discount for the portfolio almost doubles from $4 \%$ to $7 \%$ ). The presence of one single other option in a portfolio can decrease the cost of an individual ESO to the shareholders to less than a quarter of its Black-Scholes world value, and the cost of a portfolio of two options can be less than one third of its Black-Scholes world value.

This means that any approximate method for estimating the costs of ESOs which does not adjust for portfolio effects (e.g. using the Black-Scholes formula with a fixed maturity adjustment), even if it gives reasonable values for a stand-alone option, can lose significant accuracy if used to value the same option held in a portfolio.

\subsection{Portfolio composition: Moneyness effects}

In the above example, the two options were co-monotonic, so portfolio effects reduced the cost of the shorter dated option, $Y$, leaving the cost of the longer-dated option unchanged. However, we saw in Section 3 that more generally the exercise order depends on the relative strike prices and times to maturity and can change during the portfolio's life. This means the cost to the shareholders of an individual option grant to a particular executive depends on the composition of the remainder of that executive's portfolio.

\footnotetext{
${ }^{34}$ Since the options are co-monotonic, the earlier maturity option, $Y$ is always exercised first, so the cost (in the portfolio) of the ten year option is unaffected in this case.
} 


\begin{tabular}{|cc|c|c|c|c|c|} 
& & $C_{\mathcal{S}}^{Y} / C_{B S}^{Y}$ & $C^{Y} / C_{B S}^{Y}$ & $C^{Z} / C_{B S}^{Z}$ & $\left(C_{\mathcal{S}}^{Y}+C_{\mathcal{S}}^{Z}\right) /\left(C_{B S}^{Y}+C_{B S}^{Z}\right)$ & $C^{Y, Z} /\left(C_{B S}^{Y}+C_{B S}^{Z}\right)$ \\
\hline$\gamma$ & 0 & 1 & 1 & 1 & 1 & 1 \\
& 0.01 & 0.97 & 0.89 & 0.96 & 0.96 & 0.93 \\
& 0.1 & 0.68 & 0.45 & 0.65 & 0.66 & 0.56 \\
& 0.2 & 0.53 & 0.30 & 0.49 & 0.51 & 0.40 \\
\hline$\eta_{e}$ & 0.2 & 0.68 & 0.43 & 0.66 & 0.67 & 0.56 \\
& 0.4 & 0.53 & 0.30 & 0.49 & 0.51 & 0.40 \\
& 0.6 & 0.43 & 0.23 & 0.40 & 0.41 & 0.32 \\
\hline$r-q$ & 0 & 0.61 & 0.36 & 0.58 & 0.59 & 0.48 \\
& 0.03 & 0.56 & 0.32 & 0.53 & 0.55 & 0.44 \\
& 0.05 & 0.53 & 0.30 & 0.49 & 0.51 & 0.40 \\
\hline
\end{tabular}

Table 1: The ratio of option cost as a proportion of the corresponding Black-Scholes world cost for (i) $Y$ as a stand-alone, (ii) $Y$ in the portfolio with $Z$, (iii) $Z$ in a portfolio with $Y$ (=Z as stand-alone), (iv) the sum of $Y$ and $Z$ on a stand-alone basis and (v) the portfolio of $Y$ and $Z$. Option $Y$ has $K_{Y}=10, T_{Y}=5$, option $Z$ has $K_{Z}=10$ and $T_{Z}=10$. Parameters unless otherwise stated are: $S=10, r=0.10, r-q=0.05, \eta=0.4, \rho=0, \gamma=0.2$. 
Consider a portfolio of our five-year at-the-money ESO $Y$ combined with a ten-year option, $Z$, as before, but now allow $Z$ 's strike price to vary. We saw in Section 3 (Figure 6) that if $K_{Z}$ is sufficiently small, $Y$ 's exercise threshold is unaffected, since $Z$ is exercised first and $Y$ last, but that as $K_{Z}$ increases, it becomes optimal for the executive to exercise $Y$ first, at a lower threshold than the stand-alone one, when $Y$ is sufficiently close to maturity. As $K_{Z}$ increases further both the length of time when $Y$ is exercised first and also $Y$ 's exercise threshold whilst it is exercised first increase. This translates directly into an effect on $Y$ 's cost, which is shown as a function of $K_{Z}$ in the top left graph in Figure 7. The other graphs in Figure 7 show how the optimal switchover time $T^{*}$ (top right-hand graph) and cost of option $Z$ (bottom graph) vary with $K_{Z}$.

The horizontal dotted line in the top left graph represents $Y$ 's cost as a stand-alone option. This stand-alone cost is an upper bound on the cost of $Y$ when it is part of a portfolio with $Z$, which is shown by the lower, solid line. The two costs coincide for low $K_{Z}$, when $Y$ is exercised last (so $T^{*}$ in the top right graph is $T_{Y}$ ). Once $K_{Z}$ is sufficiently high that it becomes optimal to switch to exercising $Y$ first close to $Y$ 's maturity, increasing $K_{Z}$ decreases the switchover time $T^{*}$. It also decreases the cost of $Y$ because the decrease in $Y$ 's cost due to the longer period that $Y$ is exercised at its much lower, first-to-be-exercised threshold dominates the effect of the increase in the first-to-be-exercised threshold itself. For higher $K_{Z},{ }^{35}$ once $Y$ is always exercised first, only the second effect, due to the decrease in unhedgeable risk eliminated on exercise, remains, so $Y$ 's cost increases gradually with $K_{Z} \cdot{ }^{36}$ So the cost of one option in the portfolio, $Y$, varies non-monotonically with the strike price of the other option in the executive's portfolio. This is in stark contrast to a Black-Scholes world, where option values are independent of the portfolio in which they are held.

Portfolio effects also change how the cost of an option varies with its own strike price. In a Black-Scholes world, options are monotonically decreasing in their own strike price, $\frac{\partial C^{B S}}{\partial K}<0$, and this continues to hold for stand-alone options in a utility-based setting as shown by the top dashed line in the bottom graph in Figure 7. However, when $Z$ is held as part of a portfolio this

\footnotetext{
${ }^{35}$ Strictly, it is always optimal to exercise the shorter-dated option first only when the options are co-monotonic, i.e. for $K_{Z} \geq K_{Y}$ if $T_{Z} \geq T_{Y}$. However the co-monotonicity is a necessary condition only for this to be true for all possible times to maturity. In practice when the shortest time to maturity is small, if $K_{Z}$ is slightly below $K_{Y}$ the switchover time occurs long before $T_{Y}$ and has thus effectively already occurred, so $T^{*}=0$ for $K_{Z} \geq K_{Z}^{*}$ where $K_{Z}^{*}<K_{Y}$ varies with $T_{Y}$. In the example, $K_{Z}^{*}=9.23<10=K_{Y}$

${ }^{36}$ In the limit as $K_{Z} \rightarrow \infty$, there is no additional risk and $C^{Y} \rightarrow C_{\mathcal{S}}^{Y}$.
} 
is no longer necessarily true: its cost as a function of its own strike price is given by the solid line, which has a local minimum close to the strike price of the other option in the portfolio. The graph can be split into four regions. Firstly, for $K_{Z}$ sufficiently lower than the current asset price $S=10$, it is optimal for the executive to exercise $Z$ immediately, so the option cost equals its payoff. This is the case whether it is held alone or as part of a portfolio. However, immediate exercise is optimal for higher values of $K_{Z}$ (up to $K_{Z}=6.95$ in this case) when the option is held as part of a portfolio, due to the executive's lower valuation of the portfolio before exercise because of the larger associated unhedgeable risk. Secondly, for higher $K_{Z}$, it may in principle still be optimal to exercise $Z$ before $Y$, though the optimal threshold is greater than the current stock price $S^{Z}>S=10$. The cost of $Z$ thus reflects its lower threshold as the first-to-be-exercised option; this decreases as $K_{Z}$ increases due to standard decreasing moneyness considerations. Thirdly, as $K_{Z}$ increases further, at some point it becomes optimal to switch to exercising $Y$ first close to its maturity. This generates an additional effect when $K_{Z}$ increases: the decrease in switchover time $T^{*}$ increases the length of time $Z$ is exercised last, at a higher theshold, which increases $Z$ 's cost. Eventually this dominates so the cost of $Z$ has a local minimum before rising to equal its cost as a stand-alone (the fourth region when $T^{*}=0$, for $\left.K_{Z} \geq 9.23\right)$.

Finally, since the cost of the whole portfolio of ESOs granted to an individual executive is the sum of the cost of each option, evaluated as part of the portfolio: $C^{Y, Z}=C^{Y}+C^{Z}$, the portfolio cost also varies non-monotonically with the strike prices of its constituent options.

\subsection{Discounts arising from portfolio composition}

The presence of other options in the executive's portfolio reduces the cost of each individual option grant in the portfolio (as long as it does not remain the last to be exercised over the whole of its life). It is thus valued at a discount relative to its stand-alone cost, simply because it is part of a portfolio. Moreover the discount varies with the strike prices and times to maturity of the other options in the executive's portfolio. ${ }^{37}$ The "portfolio proportional discount" or proportional reduction in cost relative to the stand-alone cost due to portfolio effects can be measured as $1-\mathcal{R}_{\Pi}$ where $\mathcal{R}_{\Pi}=C^{\Pi} /\left(\sum_{i} C_{\mathcal{S}}^{\pi_{i}}\right)$ is the cost evaluated as a portfolio as a proportion

\footnotetext{
${ }^{37}$ Figure 7 shows the effects of varying the strike price of a longer-dated option. Similar effects are found varying the strike price of a shorter-dated option. Details available from the authors on request.
} 
Panel A: $\gamma=0.2, \eta=0.4, \rho=0, r=0.1, r-q=0.05$

\begin{tabular}{|c|ccc|ccc|ccc|ccc|}
\hline$T_{Z}$ & \multicolumn{3}{|c|}{$K_{Z}=8$} & \multicolumn{3}{c|}{$K_{Z}=9$} & \multicolumn{3}{c|}{$K_{Z}=10$} & \multicolumn{3}{c|}{$K_{Z}=11$} \\
\hline & $\mathcal{R}_{Y}$ & $\mathcal{R}_{Z}$ & $\mathcal{R}_{Y, Z}$ & $\mathcal{R}_{Y}$ & $\mathcal{R}_{Z}$ & $\mathcal{R}_{Y, Z}$ & $\mathcal{R}_{Y}$ & $\mathcal{R}_{Z}$ & $\mathcal{R}_{Y, Z}$ & $\mathcal{R}_{Y}$ & $\mathcal{R}_{Z}$ & $\mathcal{R}_{Y, Z}$ \\
\hline 6 & 1.000 & 0.862 & $\mathbf{0 . 9 2 3}$ & 1.000 & 0.631 & $\mathbf{0 . 8 0 4}$ & 0.557 & 1.000 & $\mathbf{0 . 7 8 2}$ & 0.620 & 1.000 & $\mathbf{0 . 8 1 0}$ \\
10 & 1.000 & 0.822 & $\mathbf{0 . 8 9 9}$ & 0.997 & 0.632 & $\mathbf{0 . 7 9 7}$ & 0.565 & 1.000 & $\mathbf{0 . 7 9 5}$ & 0.620 & 1.000 & $\mathbf{0 . 8 2 0}$ \\
15 & 1.000 & 0.798 & $\mathbf{0 . 8 8 4}$ & 0.994 & 0.636 & $\mathbf{0 . 7 9 5}$ & 0.571 & 1.000 & $\mathbf{0 . 8 0 3}$ & 0.630 & 1.000 & $\mathbf{0 . 8 2 0}$ \\
\hline
\end{tabular}

Panel B: $\gamma=0.1, \eta=0.4, \rho=0, r=0.1, r-q=0.05$

\begin{tabular}{|c|ccc|ccc|ccc|ccc|}
\hline$T_{Z}$ & \multicolumn{3}{|c|}{$K_{Z}=8$} & \multicolumn{3}{c|}{$K_{Z}=9$} & \multicolumn{3}{c|}{$K_{Z}=10$} & \multicolumn{3}{c|}{$K_{Z}=11$} \\
\hline & $\mathcal{R}_{Y}$ & $\mathcal{R}_{Z}$ & $\mathcal{R}_{Y, Z}$ & $\mathcal{R}_{Y}$ & $\mathcal{R}_{Z}$ & $\mathcal{R}_{Y, Z}$ & $\mathcal{R}_{Y}$ & $\mathcal{R}_{Z}$ & $\mathcal{R}_{Y, Z}$ & $\mathcal{R}_{Y}$ & $\mathcal{R}_{Z}$ & $\mathcal{R}_{Y, Z}$ \\
\hline 6 & 1.000 & 0.733 & $\mathbf{0 . 8 5 1}$ & 0.999 & 0.689 & $\mathbf{0 . 8 8 4}$ & 0.640 & 1.000 & $\mathbf{0 . 8 3 0}$ & 0.690 & 1.000 & $\mathbf{0 . 8 4 0}$ \\
10 & 0.999 & 0.732 & $\mathbf{0 . 8 4 7}$ & 0.987 & 0.695 & $\mathbf{0 . 8 2 6}$ & 0.650 & 1.000 & $\mathbf{0 . 8 4 0}$ & 0.690 & 1.000 & $\mathbf{0 . 8 5 0}$ \\
15 & 0.997 & 0.733 & $\mathbf{0 . 8 4 5}$ & 0.976 & 0.704 & $\mathbf{0 . 8 2 3}$ & 0.660 & 1.000 & $\mathbf{0 . 8 5 0}$ & 0.700 & 1.000 & $\mathbf{0 . 8 6 0}$ \\
\hline
\end{tabular}

Panel C: $\gamma=0.2, \eta=0.6, \rho=0, r=0.1, r-q=0.05$

\begin{tabular}{|c|ccc|ccc|ccc|ccc|}
\hline$T_{Z}$ & \multicolumn{3}{|c|}{$K_{Z}=8$} & \multicolumn{3}{c|}{$K_{Z}=9$} & \multicolumn{3}{c|}{$K_{Z}=10$} & \multicolumn{3}{c|}{$K_{Z}=11$} \\
\hline & $\mathcal{R}_{Y}$ & $\mathcal{R}_{Z}$ & $\mathcal{R}_{Y, Z}$ & $\mathcal{R}_{Y}$ & $\mathcal{R}_{Z}$ & $\mathcal{R}_{Y, Z}$ & $\mathcal{R}_{Y}$ & $\mathcal{R}_{Z}$ & $\mathcal{R}_{Y, Z}$ & $\mathcal{R}_{Y}$ & $\mathcal{R}_{Z}$ & $\mathcal{R}_{Y, Z}$ \\
\hline 6 & 1.000 & 0.834 & $\mathbf{0 . 9 1 0}$ & 1.000 & 0.602 & $\mathbf{0 . 7 9 2}$ & 0.527 & 1.000 & $\mathbf{0 . 7 6 6}$ & 0.593 & 1.000 & $\mathbf{0 . 7 9 1}$ \\
10 & 1.000 & 0.809 & $\mathbf{0 . 8 9 5}$ & 0.999 & 0.605 & $\mathbf{0 . 7 8 9}$ & 0.535 & 1.000 & $\mathbf{0 . 7 7 6}$ & 0.596 & 1.000 & $\mathbf{0 . 7 9 9}$ \\
15 & 1.000 & 0.799 & $\mathbf{0 . 8 8 9}$ & 0.998 & 0.607 & $\mathbf{0 . 7 8 8}$ & 0.536 & 1.000 & $\mathbf{0 . 7 7 9}$ & 0.596 & 1.000 & $\mathbf{0 . 8 0 2}$ \\
\hline
\end{tabular}

Panel D: $\gamma=0.2, \eta=0.4, \rho=0, r=0.05, r-q=0.05$

\begin{tabular}{|c|ccc|ccc|ccc|ccc|}
\hline$T_{Z}$ & \multicolumn{3}{|c|}{$K_{Z}=8$} & \multicolumn{3}{c|}{$K_{Z}=9$} & \multicolumn{3}{c|}{$K_{Z}=10$} & \multicolumn{3}{c|}{$K_{Z}=11$} \\
\hline & $\mathcal{R}_{Y}$ & $\mathcal{R}_{Z}$ & $\mathcal{R}_{Y, Z}$ & $\mathcal{R}_{Y}$ & $\mathcal{R}_{Z}$ & $\mathcal{R}_{Y, Z}$ & $\mathcal{R}_{Y}$ & $\mathcal{R}_{Z}$ & $\mathcal{R}_{Y, Z}$ & $\mathcal{R}_{Y}$ & $\mathcal{R}_{Z}$ & $\mathcal{R}_{Y, Z}$ \\
\hline \multirow{2}{*}{6} & 1.000 & 0.710 & $\mathbf{0 . 8 4 0}$ & 0.998 & 0.669 & $\mathbf{0 . 8 2 3}$ & 0.630 & 1.000 & $\mathbf{0 . 8 1 0}$ & 0.666 & 1.000 & $\mathbf{0 . 8 3 1}$ \\
10 & 0.998 & 0.711 & $\mathbf{0 . 8 3 3}$ & 0.980 & 0.678 & $\mathbf{0 . 8 1 1}$ & 0.640 & 1.000 & $\mathbf{0 . 8 3 0}$ & 0.671 & 1.000 & $\mathbf{0 . 8 4 5}$ \\
15 & 0.995 & 0.711 & $\mathbf{0 . 8 2 9}$ & 0.948 & 0.696 & $\mathbf{0 . 8 0 3}$ & 0.640 & 1.000 & $\mathbf{0 . 8 4 0}$ & 0.675 & 1.000 & $\mathbf{0 . 8 5 5}$ \\
\hline
\end{tabular}

Panel E: $\gamma=0.2, \eta=0.4, \rho=0, r=0.1, r-q=0.03$

\begin{tabular}{|c|ccc|ccc|ccc|ccc|}
\hline$T_{Z}$ & \multicolumn{3}{|c|}{$K_{Z}=8$} & \multicolumn{3}{c|}{$K_{Z}=9$} & \multicolumn{3}{c|}{$K_{Z}=10$} & \multicolumn{3}{c|}{$K_{Z}=11$} \\
\hline & $\mathcal{R}_{Y}$ & $\mathcal{R}_{Z}$ & $\mathcal{R}_{Y, Z}$ & $\mathcal{R}_{Y}$ & $\mathcal{R}_{Z}$ & $\mathcal{R}_{Y, Z}$ & $\mathcal{R}_{Y}$ & $\mathcal{R}_{Z}$ & $\mathcal{R}_{Y, Z}$ & $\mathcal{R}_{Y}$ & $\mathcal{R}_{Z}$ & $\mathcal{R}_{Y, Z}$ \\
\hline 6 & 1.000 & 0.900 & $\mathbf{0 . 9 4 4}$ & 1.000 & 0.640 & $\mathbf{0 . 8 0 9}$ & 0.570 & 1.000 & $\mathbf{0 . 7 9 0}$ & 0.640 & 1.000 & $\mathbf{0 . 8 1 0}$ \\
10 & 1.000 & 0.866 & $\mathbf{0 . 9 2 3}$ & 0.998 & 0.648 & $\mathbf{0 . 8 0 6}$ & 0.580 & 1.000 & $\mathbf{0 . 8 0 0}$ & 0.640 & 1.000 & $\mathbf{0 . 8 2 0}$ \\
15 & 1.000 & 0.848 & $\mathbf{0 . 9 1 2}$ & 0.997 & 0.654 & $\mathbf{0 . 8 0 6}$ & 0.580 & 1.000 & $\mathbf{0 . 8 1 0}$ & 0.640 & 1.000 & $\mathbf{0 . 8 3 0}$ \\
\hline
\end{tabular}

Table 2: The ratio of the option cost calculated on a portfolio basis to the (sum of the) stand-alone $\operatorname{cost}(\mathrm{s}): \mathcal{R}_{Y}=C^{Y} / C_{\mathcal{S}}^{Y}, \mathcal{R}_{Z}=C^{Z} / C_{\mathcal{S}}^{Z}$ in italics and $\mathcal{R}_{Y, Z}=C^{Y, Z} /\left(C_{\mathcal{S}}^{Y}+C_{\mathcal{S}}^{Z}\right)$ in bold. Option $Y$ has $K_{Y}=10, T_{Y}=5$. The Table varies $K_{Z}$ and $T_{Z}$. Parameters unless otherwise stated are: $S=10$, $r=0.10, r-q=0.05, \eta=0.4, \rho=0, \gamma=0.2$. 
of the (sum of) stand-alone cost(s). For a portfolio of two or more unexercised options this is always non-zero, i.e. $\mathcal{R}_{\Pi}<1.38$

The bottom right graph in Figure 7 plots $\mathcal{R}_{Y, Z}$ vs $K_{Z}$ for our two option portfolio of $Y$ with longer dated $Z$. The discount for the portfolio as a whole arises from option $Z$ when $K_{Z}$ is sufficiently low, from option $Y$ when $K_{Z}$ is high (e.g. $K_{Z}>K_{Y}$ ), and from both options when $K_{Z}$ is slightly lower than $K_{Y}$ (in the switching region $D$ from Figure 5). The proportional discount for the portfolio as a whole reaches a maximum value (of over 19\%) for some $K_{Z}$ in the switching region, $D$, but remains above $10 \%$ for a wide range of $K_{Z} \cdot{ }^{39}$

Table 2 shows, for each option, $Y$ and $Z$, and for the portfolio as a whole, $Y+Z$, the portfolio cost as a proportion of the (sum of) stand-alone $\operatorname{cost}(\mathrm{s}), \mathcal{R}_{Y} \equiv C^{Y} / C_{\mathcal{S}}^{Y}$ etc., for different strike prices and maturities $K_{Z}$ and $T_{Z}$. Panel A considers the same parameter values as in the earlier example; panels B - E show the effect of varying each key parameter (the executive's risk aversion, $\gamma$, the stock's (unhedgeable) risk, $\eta$, the risk-free rate, $r$ and the stock's dividend yield, via $r-q$. For each combination of $\left(K_{Z}, T_{Z}\right)$, either it is always optimal to exercise the shorterdated option, $Y$, first, so $\mathcal{R}_{Y}<1$ and $\mathcal{R}_{Z}=1$, or always optimal to exercise the longer dated option, $Z$, first so $\mathcal{R}_{Y}=1, \mathcal{R}_{Z}<1$, or the optimal exercise order changes, so both $\mathcal{R}_{Y}, \mathcal{R}_{Z}<1$. In the Table, since $T_{Z}>T_{Y}$, these possibilities correspond to regions $\mathrm{B}, \mathrm{F}$ and $\mathrm{D}$ in Figure 5.

For low $K_{Z}$ it is optimal for the executive to exercise $Z$ immediately, so the cost of $Z$ as part of the portfolio equals its payoff on exercise. If $Z$ 's optimal exercise threshold on a stand-alone basis is higher than the current stock price, $S_{0}$, then the portfolio discount is entirely due to the time value of $Z$ 's cost as a stand-alone option. This increases as $K_{Z}$, and hence the difference between $Z$ 's stand-alone threshold and $S_{0}$ increases. Alternatively, if $K_{Z}$ is sufficiently high that the portfolio discount is entirely due to $Y$ 's lower threshold as the first-to-be-exercised option, then increases in $K_{Z}$ reduce $Z$ 's moneyness and hence reduce the marginal unhedgeable risk on exercise of $Y$, reducing the discount. ${ }^{40}$ For $K_{Z}$ in the intermediate region where it is initially optimal to exercise $Z$ first, but not immediately, and the optimal exercise order may change during the options' lives, a number of effects arise when $K_{Z}$ increases. The increase in the

\footnotetext{
${ }^{38}$ At any time all but one of the options in a portfolio are exercised at lower thresholds and thus have lower costs than if held as stand-alone options.

${ }^{39} \mathcal{R}_{\Pi}=1$ only for low $K_{Z}$ where $Z$ is so far in-the-money (recall $S=10$ ) that it is optimal to exercise $Z$ immediately.

${ }^{40}$ This dominates over the additional effect of the increased weight of $Y$ in the portfolio due to the reduction in $Z$ 's moneyness and hence cost.
} 
marginal unhedgeable risk reduction on exercise of the first-to-be-exercised option continues to reduce the portfolio discount, but the increase in $K_{Z}$ also changes both the length of time for which $Y$ is exercised first and the relative weights of $Y$ and $Z$ in the portfolio. Which effect dominates depends on the parameter values. Overall the proportional discount for the portfolio as a whole is maximized within this region where the options are non co-monotonic and initially the longer dated option is exercised first. Moreover, the further away (in $(K, T)$ space) from the switching region, (e.g. higher $K_{Z}>K_{Y}$ or lower $K_{Z} \ll K_{Y}$ ), the lower the proportional discount.

The Table shows that portfolio effects can reduce shareholder costs for the portfolio as a whole by more than $20 \%$ relative to the costs of the options as stand-alones, and by over $40 \%$ for individual options. Moreover, the proportional discount remains significant for a wide range of portfolios ( $K$ 's and $T$ 's) and parameter values. The portfolio proportional discount is larger for firms with more risk-averse executives, with high idiosyncratic volatility and low dividend yield, and when the risk-free rate is high, and for executives with portfolios where earlier-maturity options have higher strike prices ${ }^{41}$.

Firms should thus recognize that evaluating the cost of each ESO individually overstates the total cost to the shareholders of a portfolio of options granted to the same executive. The inherent non-linearity induced by portfolio considerations means the cost of an ESO portfolio needs to be calculated as a whole. Moreover, the costs of individual ESOs depend on the portfolio they are a part of, and so may change if the composition of the executive's portfolio changes. This raises the question of how a firm should evaluate the cost of a new option grant.

\subsection{Incremental cost of new options}

If the executive receives an additional option grant, portfolio size and total unhedgeable risk increase. The overall portfolio cost increases, but the discount relative to the sum of the standalone costs also increases. Consider the effect of an unanticipated new grant of an option, $Z$, to an executive with an existing portfolio of options (consisting of option $Y$ ). Before option $Z$ is granted, the cost to the shareholders of the executive's portfolio would be given by $C_{\mathcal{S}}^{Y}$, the cost of option $Y$ as a stand-alone. After option $Z$ is granted, the cost of the portfolio would be $C^{Y}+C^{Z}$, where both costs take into account the presence of the other option in the portfolio.

\footnotetext{
${ }^{41}$ So the optimal exercise order changes and both options will optimally be exercised first in different time periods.
} 
Thus the incremental cost of the new grant to the shareholders is $C_{Z \mid Y}^{\text {marginal }} \equiv C^{Y}+C^{Z}-C_{\mathcal{S}}^{Y}$. This is plotted in Figure 8 for different strike prices for the newly granted option, $K_{Z}$, assuming option $Y$ is currently at-the-money. Since $C^{Y}$ and $C^{Z}$ vary non-monotonically with respect to $K_{Z}$, as shown in Figure 7, the incremental cost of option $Z$ also depends on $K_{Z}$ in a more complex way than would be expected in a Black-Scholes world. In particular, it is no longer monotonically decreasing with respect to $K_{Z}$ : there is a local minimum incremental cost at a strike price slightly smaller than $K_{Y}$. Equivalently, the local minimum incremental cost for the new option occurs if the option is granted slightly in-the-money, and the incremental cost of the new option is less sensitive to changes in its strike price when it is granted close to the money.

The left-hand graph in Figure 9 shows $\mathcal{R}_{\Pi}$ or (1 - "portfolio proportional discount") for the incremental cost of $Z$ vs $K_{Z}$ assuming option $Y$ is at-the-money. Note as long as it is optimal to hold both options, the proportional discount is non-zero. It has a similar shape to the graph for the portfolio as a whole, but at a lower level. ${ }^{42}$ The maximum proportional discount occurs if the new option is granted slightly in-the-money and remains above $20 \%$ for most choices of strike price and time to maturity.

It is common for firms to grant all ESOs at-the-money with the same time to maturity (10 years)(Carpenter et al (2012)). The right-hand graph in Figure 9 shows how $\mathcal{R}_{\Pi}$ and hence the portfolio proportional discount varies with the stock price for an incremental option granted atthe-money with 10 years to maturity to an executive who already holds an ESO with strike price 10 and five years to maturity. The proportional discount is also non-monotonic with respect to $K_{Z}$. It is non-zero as long as it is not optimal to exercise the existing option, $Y$, immediately, and over $10 \%$ for stock prices at least $30 \%$ in- and out-of-the-money for the existing option, $Y$.

So firms also need to recognize that the cost of the same option grant to otherwise identical executives, with in particular the same level of risk aversion, will not be the same if the portfolio of other options they hold differs. The magnitude of the portfolio proportional discounts suggest any approximate method for evaluating the cost of ESOs needs to be flexible enough to take these portfolio considerations into account. Increasing the size of the existing portfolio (adding options) will decrease the cost of making a new option grant to the executive. So the cost to the shareholders of an option grant to an executive is highest when the executive has no outstanding

\footnotetext{
${ }^{42}$ This is unsurprising since both incorporate the reductions in cost of all options in the portfolio due to the additional option grant in the numerator but the denominator is smaller for the individual option than for the portfolio as a whole.
} 
unexercised ESOs.

\section{Robustness}

\subsection{Larger portfolios}

Up to this point, we have only considered in detail portfolios consisting of two options. As the number of options increases, the complexity of the numerical solution increases significantly because of the number of potential exercise combinations which need to be considered, thus we leave detailed investigation of optimal exercise strategies for larger portfolios for further work. However, many of the results for the two-option case will carry over to larger portfolios, potentially with even greater effects. This is because of the common underlying cause of the results, that the effect of unhedgeable risk increases non-linearly with portfolio size. This implies that thresholds for a given option when it is $(n+1)$ st-to-last to be exercised are distinctly lower than the threshold for the same option when one option is removed from the remainder of the portfolio, so it is the $n$ th-to-last to be exercised, because the decrease in unhedgeable risk associated with the option exercise is greater, the larger the remaining portfolio. This means that the threshold at which an executive exercises her first option decreases when further options are added to her portfolio. ${ }^{4344}$

Exercise thresholds for a particular option in the larger portfolio can exhibit the same characteristics as in the two-option case: the threshold can increase, even at an increasing rate, as time-to-maturity decreases, and will jump if its position in the optimal exercise order changes. However the jumps no longer affect all options in the portfolio: the exercise threshold for options not involved in a particular switch in the exercise order remain continuous across the switching date. An example of exercise thresholds for a portfolio of three options is given in Figure 10. Depending on the combinations of strike prices and maturity dates, there can be multiple switches in the exercise order and jumps in exercise thresholds. For portfolios with co-monotonic strike prices and maturities, there are still no jumps and the exercise order remains unchanged

\footnotetext{
${ }^{43}$ If the new option is the first-to-be-exercised, its threshold is automatically lower than the lowest threshold of the existing options. If at some time it is not the first-to-be-exercised, thresholds for all options exercised before it, including the first-to-be-exercised, are reduced since the portfolio remaining after their exercise now includes the new option.

${ }^{44}$ We know for a single grant of identical options, as the number of options becomes infinite, the individual exercise thresholds will limit to the option strike $K$, see Henderson and Hobson (2011).
} 
throughout the portfolio's life.

In general the exercise order depends on the relationship between the strike prices and maturities of options in the portfolio. Holding all else equal, options with higher strike prices and longer times to maturity are exercised later; where these effects conflict, differences in strike price are more likely to dominate when all the times to maturity are long but an option with a higher strike price but shorter maturity can be exercised out of strike-price order when its time to maturity is short since its thresholds decrease rapidly as its time-to-maturity decreases to zero.

These effects carry through to shareholder costs. At most one option in a portfolio has the same cost as its stand-alone cost (if it is always last-to-be-exercised); costs for all other options are lower because they are no longer exercised at their stand-alone, equivalently lastto-be-exercised thresholds. Moreover, the cost of each option in a portfolio is affected by its relationship with the strike prices and maturities of all the other options in the portfolio. As a function of its strike price, the shareholder cost of a new option can have multiple local minima. ${ }^{45}$ Costs of all existing options will also depend non-linearly on the strike price of the new option, generally with a similar shape to the graph in the two-option case (the top left graph in Figure 7). Hence the incremental cost to the shareholders of a new option grant will also be non-linear with potentially multiple local minima.

When an option portfolio's size is increased by the addition of an option, the cost of the overall portfolio to the shareholders will increase, but by much less than the stand-alone cost of the new option. The proportional portfolio discount for new options, or equivalently the difference between the incremental cost of a new option taking account of portfolio effects and its cost as a stand-alone will increase. ${ }^{46}$

\footnotetext{
${ }^{45}$ If it has a sufficiently low strike price, the new option will be exercised first, say $(n+1)$ st to last. However, as its strike price increases, its position in the exercise order changes to $n$ th-to-last, $(n-1)$ st-to-last, etc.. Eventually, for high enough strike prices, it becomes the last to be exercised. The exercise threshold and shareholder cost of an option exercised later in the exercise order increases, because of the lower level of unhedgeable risk associated with the exercise decision. However the cost of an option with a given place in the exercise order decreases with its strike price. Together this may lead to a local minimum for each switch in the exercise order, as in the two-option case.

${ }^{46}$ On the addition of an option to an existing portfolio, the costs of options in the existing portfolio which will now be exercised after the new option are unaffected by its presence; however any options now exercised before the new option at any time will have a lower cost. If the existing portfolio increases in size and, when added, the new option is still exercised last, its threshold and hence cost will be lower. If it is not exercised last in the larger
} 
Overall then, portfolio effects on both exercise thresholds and costs increase when options are added to an ESO portfolio, but the nature of the effects remains broadly the same as in the two option case we considered in detail in Sections 3 and 4.

\subsection{Vesting, employment risk and performance-based compensation}

We finish by discussing several issues concerning the robustness of our results to inclusion of vesting, employment risk, and performance-based compensation.

In practice, ESOs commonly have vesting restrictions in order to tie the executive to the company and provide incentives. ${ }^{47}$ Very few of the works taking unhedgeble risks into account also consider the impact of time-vesting ${ }^{48}$ however, Leung and Sircar (2009) find shareholder costs increase with the vesting period. In our framework, vesting restrictions would not alter the optimal exercise ordering once the options had vested, and it would not change the thresholds at all if the options vested at the same time. ${ }^{49}$

Executives may be forced to exercise or forfeit options if their employment is terminated. Leung and Sircar (2009) and Carpenter et al (2010) consider exogenous job termination risk and find it reduces the exercise threshold and shareholder costs. We do not include job termination risk in our setting as it would not alter the ordering of exercise of options within a portfolio. ${ }^{50}$ Importantly, its effect would be in the same direction as the impact of portfolio effects from unhedgeable risk and thus inclusion of employment risk would reduce shareholder costs further.

Although our focus has been on portfolios of standard ESOs, many of our conclusions will be relevant also for performance-based compensation where there are additional performancevesting or payout conditions based on stock price, accounting performance or industry comparisons (see Bettis et al (2010), Johnson and Tian (2000)).

combined portfolio, then at least one option in the portfolio is exercised at a lower threshold than it would be in the combined portfolio before the increase in size, lowering the incremental cost.

${ }^{47}$ See Kole (1997) for a comprehensive analysis.

${ }^{48}$ Carpenter et al (2010) incorporate vesting into their shareholder costs (but not their analysis of exercise thresholds) but do not compare to costs of an equivalent option without vesting.

${ }^{49} \mathrm{We}$ anticipate that if the vesting times are different, there will be a small impact on some of the thresholds of options which have vested when other options remain unvested, but we do not believe this will alter our main findings or have a significant quantitative effect.

${ }^{50}$ Incorporation of exogenous job termination would result in a free boundary problem of reaction-diffusion type. Our transformation to the heat equation (see Appendix) would not apply and we would need to use alternative methods. 


\section{Conclusion}

In this paper we have extended the literature on utility based valuation of ESOs to model the behavior of an executive with a portfolio of options with various strikes and time to maturity. Since unhedgeable risk varies non-linearly with portfolio size and composition, the executive's exercise strategy and thus also the shareholder cost of an option held as part of a portfolio depend on the remainder of the executive's ESO portfolio. These portfolio effects are important - lowering both the moneyness required for exercise and the shareholder cost of most options in a portfolio. In fact, the proportional reduction in cost relative to stand-alone options even in the case of portfolios of only two options can be over $40 \%$ for individual options and $20 \%$ for portfolios. In contrast to a risk-neutral setting, both exercise thresholds and costs depend on an option's position in the optimal exercise order: when the exercise order switches, the thresholds of options exercised earlier (later) jump down (up). Given these dependencies, the company should re-evaluate the costs of all outstanding ESOs each time they give a new grant of options to their executives - as executives may alter the moneyness and order at which they exercise their existing options when they receive a new grant.

We use the model to explain several empirical findings in the literature - which options are attractive to exercise first, how exercise can be induced by a new grant, and the prevalence of early exercise. This highlights the importance of including portfolio effects in an empirical study of executive exercise behavior and accounting for such effects in any measure based on exercise behavior.

Whilst there is scope for further work on ESO portfolios, this paper has set out the key principles of how portfolio effects impact ESO thresholds and costs. Firstly, for individual options in portfolios, the option's position in the portfolio's optimal exercise order is the new key factor determining both an option's exercise threshold and its cost within the portfolio. Secondly, for portfolios as a whole, what matters is the overall strength of the portfolio interaction effects. This determines the discount for the portfolio as a whole and the moneyness on exercise of the first option to be exercised in the portfolio. It depends on both the size and the overall composition of the portfolio, increasing when an option is added to a portfolio, and reaching a maximum when the strike prices of longer-dated options are slightly less than those of shorter-dated options within the portfolio. Finally, the relevant cost of an option grant is its incremental cost, which depends on the composition of the portfolio it is being combined with, and which is always lower than the option's stand-alone cost, since it reflects the greater additional unhedgeable risk 
associated with the option's addition to the executive's existing portfolio.

\section{$7 \quad$ Appendix}

\subsection{The Model}

We first use separation of variables and a power transformation ${ }^{51}$ via

$$
V^{\Pi}(u, w, s)=\mathcal{M}(u, w, \tilde{T}) H^{\Pi}(u, s)^{1 /\left(1-\rho^{2}\right)}
$$

to restate (4), (5) and (9) as

$$
\begin{gathered}
H^{\Pi}\left(u, S_{u}\right)^{1 /\left(1-\rho^{2}\right)} \leq \min _{\pi \in \Pi}\left\{e^{\left.-\gamma\left(1-\rho^{2}\right)\left(S_{u}-K_{\pi}\right)^{+} e^{r(\tilde{T}-u)} H^{\Pi \backslash\{\pi\}}\left(u, S_{u}\right)^{1 /\left(1-\rho^{2}\right)}\right\}}\right. \\
\frac{\partial H^{\Pi}}{\partial t}+\tilde{\mathcal{L}} H^{\Pi} \geq 0
\end{gathered}
$$

where the differential operator $\tilde{\mathcal{L}}$ is defined by

$$
\tilde{\mathcal{L}}=\frac{\eta^{2} s^{2}}{2} \frac{\partial^{2}}{\partial s^{2}}+(r-q) s \frac{\partial}{\partial s}
$$

and

$$
\tau^{(|\Pi|)}=\inf \left\{t \leq u \leq T_{\min }: H^{\Pi}\left(u, S_{u}\right)=\min _{\pi \in \Pi}\left\{e^{-\gamma\left(1-\rho^{2}\right)\left(S_{u}-K_{\pi}\right)^{+} e^{r(\tilde{T}-u)}} H^{\Pi \backslash\{\pi\}}\left(u, S_{u}\right)\right\}\right\} .
$$

The boundary conditions are given by $H^{\Pi}(u, 0)=1$ and

$$
\forall\{\pi\} \in \Pi ; \quad \frac{H^{\Pi}\left(T_{\pi}, S_{T_{\pi}}\right)}{H^{\Pi \backslash\{\pi\}}\left(T_{\pi}, S_{T_{\pi}}\right)}=\frac{M\left(T_{\pi}, X_{T_{\pi}}+\left(S_{T_{\pi}}-K_{\pi}\right), \tilde{T}\right)}{M\left(T_{\pi}, X_{T_{\pi}}, \tilde{T}\right)}=e^{-\gamma\left(1-\rho^{2}\right)\left(S_{T_{\pi}}-K_{\pi}\right)^{+} e^{r\left(\tilde{T}-T_{\pi}\right)}}
$$

and we have $H^{\emptyset}\left(u, S_{u}\right)=1$.

We see in (16) that associated with each $H^{\Pi}\left(u, S_{u}\right)$ there is a free boundary

$$
S^{(|\Pi|)}(u)=\inf \left\{s \geq 0: H^{\Pi}(u, s)=\min _{\pi \in \Pi}\left\{e^{-\gamma\left(1-\rho^{2}\right)\left(s-K_{\pi}\right)^{+} e^{r(\tilde{T}-u)}} H^{\Pi \backslash\{\pi\}}(u, s)\right\} ; u \in\left[t, T_{\min }\right]\right\}
$$

which represents the exercise boundary for the next option when the options $\Pi$ remain. As with standard American options (see (26)), the optimal exercise times can be represented as

$$
\tau^{(|\Pi|)}=\inf \left\{t \leq u \leq T_{\min }: S_{u}=S^{(|\Pi|)}(u)\right\}
$$

\footnotetext{
${ }^{51}$ The separation of variables is simply the observation that wealth factors out under exponential utility. We follow many authors who employ the power transformation in pricing of European and American options under exponential utility, for example Henderson (2005).
} 


\section{Subjective Value}

We can use the definition of the subjective value to the executive in (12) to re-derive the free boundary problem as follows: $p^{\Pi}(t, s)$ solves

$$
\begin{aligned}
& \frac{\partial p^{\Pi}}{\partial t}+\tilde{\mathcal{L}} p^{\Pi}-r p^{\Pi}-\frac{1}{2} \gamma\left(1-\rho^{2}\right) \eta^{2} s^{2} e^{r(\tilde{T}-t)}\left(\frac{\partial p^{\Pi}}{\partial s}\right)^{2} \leq 0 \\
& p^{\Pi}(t, s) \geq \max _{\pi \in \Pi}\left\{\left(s-K_{\pi}\right)^{+}+p^{\Pi \backslash\{\pi\}}(t, s)\right\}
\end{aligned}
$$

with boundary conditions $p^{\Pi}(t, 0)=0$ and

$$
\forall\{\pi\} \in \Pi ; \quad p^{\Pi}\left(T_{\pi}, S_{T_{\pi}}\right)-p^{\Pi \backslash\{\pi\}}\left(T_{\pi}, S_{T_{\pi}}\right)=\left(S_{T_{\pi}}-K_{\pi}\right)^{+} .
$$

\section{Shareholder Costs}

We can use the optimal exercise threshold for each option $S^{\pi}(u) ; \pi \in \Pi$ as input into the shareholder costs. To compute the shareholder cost of a portfolio $\Pi$, we compute the $\operatorname{cost} C^{\pi}$ for each option $\pi \in \Pi$ and sum over all options. Each $C^{\pi}$ satisfies for $s \leq S^{\pi}(t)$

$$
\frac{\partial C^{\pi}}{\partial t}+\tilde{\mathcal{L}} C^{\pi}=0
$$

with boundary conditions $\forall \pi \in \Pi, C^{\pi}(u, 0)=0, C^{\pi}\left(T_{\pi}, S_{T_{\pi}}\right)=\left(S_{T_{\pi}}-K_{\pi}\right)^{+}$and $C^{\pi}\left(u, S^{\pi}(u)\right)=$ $\left(S^{\pi}(u)-K_{\pi}\right)^{+} ; u<T_{\pi}$.

\section{Black Scholes}

Consider a single call option $Y$ with strike $K_{Y}$, maturity $T_{Y}$ and assume we price under the Black Scholes model. The value $V^{B S}\left(u, S_{u}\right)$ of holding the American call solves the well known variational inequalities:

$$
\begin{gathered}
V^{B S}\left(u, S_{u}\right) \geq\left(S_{u}-K_{Y}\right)^{+} \\
\frac{\partial V^{B S}}{\partial t}+\tilde{\mathcal{L}} V^{B S}-r V^{B S} \leq 0 .
\end{gathered}
$$

Boundary conditions are given by $V^{B S}(u, 0)=0$ and $V^{B S}\left(T_{Y}, S_{T_{Y}}\right)=\left(S_{T_{Y}}-K_{Y}\right)^{+}$. The optimal exercise time $\tau^{B S}$ is defined by

$$
\tau^{B S}=\inf \left\{t \leq u \leq T_{Y}: V^{B S}\left(u, S_{u}\right)=\left(S_{u}-K_{Y}\right)^{+}\right\}
$$

which defines an exercise boundary

$$
S^{B S}(u)=\inf \left\{s \geq 0: V^{B S}(u, s)=\left(s-K_{Y}\right)^{+} ; u \in\left[t, T_{Y}\right]\right\}
$$

and thus

$$
\tau^{B S}=\inf \left\{t \leq u \leq T_{Y}: S_{u}=S^{B S}(u)\right\}
$$




\subsection{Numerical Method}

\section{Utility Model}

We first transform to work with the heat equation which is useful for comparison to Black Scholes and to simplify the coding. Define new variables $x \in \mathbb{R}$ and $\tau \in\left[0,0.5 \eta^{2} \tilde{T}\right]$ via $s=\delta e^{x}$ and $t=\tilde{T}-\tau / 0.5 \eta^{2}$, where $\delta$ is a constant. Write $H^{\Pi}\left(\tilde{T}-\tau / 0.5 \eta^{2}, \delta e^{x}\right)=\delta u^{\Pi}(x, \tau) e^{-\xi x-\xi^{2} \tau}$ where $\xi=0.5\left((r-q) / 0.5 \eta^{2}-1\right)$. Then (14) is given by

$$
\partial u^{\Pi} / \partial \tau-\partial^{2} u^{\Pi} / \partial x^{2} \leq 0
$$

and (13) becomes

$$
u^{\Pi}(x, \tau) \leq g^{\Pi}(x, \tau)
$$

with

$$
g^{\Pi}(x, \tau)=\min _{\pi \in \Pi}\left\{e^{-\gamma\left(1-\rho^{2}\right)\left(\delta e^{x}-K_{\pi}\right)^{+} e^{r \tau / 0.5 \eta^{2}}} u^{\Pi \backslash\{\pi\}}(x, \tau)\right\} .
$$

The boundary conditions are $u^{\Pi}\left(x_{\min }, \tau\right)=\frac{1}{\delta} e^{\delta x_{\min }+\delta^{2} \tau}$ and

$$
\forall\{\pi\} \in \Pi ; \quad \frac{u^{\Pi}\left(x, \tau_{\pi}\right)}{u^{\Pi \backslash\{\pi\}}\left(x, \tau_{\pi}\right)}=e^{-\gamma\left(1-\rho^{2}\right)\left(\delta e^{x}-K_{\pi}\right)^{+} e^{r \tau_{\pi} / 0.5 \eta^{2}}}
$$

where $\tau_{\pi}=0.5 \eta^{2}\left(\tilde{T}-T_{\pi}\right)$.

To solve (27)-(28) subject to the boundary conditions, we use a Crank Nicolson finite difference method on a uniform grid. The free boundary constraint (28) is enforced by a a projected successive over relaxation algorithm (PSOR), see Wilmott et al (1995) for similar schemes in a Black Scholes framework. We restrict the domain $\mathbb{R} \times\left[0,0.5 \eta^{2} \tilde{T}\right]$ to a finite domain $\left\{(x, \tau): x_{\min } \leq x \leq x_{\max }, 0 \leq \tau \leq 0.5 \eta^{2} \tilde{T}\right\}$. We introduce a uniform grid with nodes $\left\{\left(x_{\min }+j \Delta x, n \Delta \tau\right): j=0,1, \ldots, j_{\max }, n=0,1, \ldots, n_{\max }\right\}$ with grid spacings $\Delta x=\left(x_{\max }-x_{\min }\right) / j_{\max }, \Delta \tau=0.5 \eta^{2} \tilde{T} / n_{\max }$. We apply discrete approximations $U_{j}^{n} \approx$ $u^{A}\left(x_{\min }+j \Delta x, n \Delta \tau\right)$ and approximate the derivatives by:

$$
\begin{gathered}
\frac{\partial u^{\Pi}(x, \tau)}{\partial \tau} \approx \frac{U_{j}^{n+1}-U_{j}^{n}}{\Delta \tau} \\
\frac{\partial^{2} u^{\Pi}(x, \tau)}{\partial x^{2}} \approx \frac{1}{2}\left(\frac{U_{j+1}^{n}-2 U_{j}^{n}+U_{j-1}^{n}}{(\Delta x)^{2}}+\frac{U_{j+1}^{n}-2 U_{j}^{n}+U_{j-1}^{n}}{(\Delta x)^{2}}\right)
\end{gathered}
$$

giving

$$
U_{j}^{n+1}-\frac{1}{2} a\left(U_{j-1}^{n+1}-2 U_{j}^{n+1}+U_{j+1}^{n+1}\right)=U_{j}^{n}+\frac{1}{2} a\left(U_{j-1}^{n}-2 U_{j}^{n}+U_{j+1}^{n}\right)
$$


where $a=\Delta \tau /(\Delta x)^{2}$. We solve (29) (together with the discretized boundary conditions) backward in time using the PSOR algorithm to compute the optimal boundary $s^{*}=\delta e^{x_{m i n}+j^{*} \Delta x}$ at each time step $n \Delta \tau$ by finding the minimum index $j=j^{*}$ such that $U_{j^{*}}^{n}=g^{\Pi}\left(x_{\min }+j^{*} \Delta x, n \Delta \tau\right)$. Shareholder Costs

Similar transformations and approximations are used to solve (22) together with the given boundary conditions. (In this case there is no free boundary, so we do not require the PSOR.)

\section{Black Scholes}

Each option in the portfolio can be treated separately under the Black Scholes model since prices are linear. It's price solves (23) and (24) together with the given boundary conditions. We follow the same transformation to the heat equation and numerical finite difference scheme as outlined earlier.

\section{Robustness}

We perform various robustness checks for the utility model algorithm. We experimented with the size of the grid versus speed and use $j_{\max }=2000, n_{\max }=9000, x_{\min }=-3$ and $x_{\max }=3$. Using the utility model algorithm gives thresholds and values that converge to the correct Black Scholes quantities (calculated from (23) and (24)) as $\gamma \rightarrow 0$ or $\rho \rightarrow 1$. For large maturities and identical strikes, we can recover the time-homogeneous thresholds and values derived in explicitly by the perpetual approximation of Grasselli and Henderson (2009). 


\section{References}

Armstrong C.S., Jagolinzer A.D. and D.F. Larcker, 2007, "Timing of Employee Stock Option Exercises and the Valuation of Stock Option Expense", Rock Center for Corporate Governance Working Paper No. 34. Available at SSRN: http://ssrn.com/abstract $=905280$.

Bergstresser D.B. and T. Philippon, 2006, "CEO incentives and earnings management", Journal of Financial Economics, 80, 511-529.

Bettis J.C., Bizjak J.M. and Kalpathy., 2011, "Why do insiders hedge their ownership? An empirical examination", Working paper. Available at: SSRN: http://ssrn.com/abstract=1364810.

Bettis J.C., Bizjak J.M. and Lemmon M.L., 2005, "Exercise behavior, valuation and the incentive effects of employee stock options", Journal of Financial Economics, 76, 445-470.

Bettis J.C., Bizjak J.M., Coles J. and Kalpathy S., 2010, "Stock and option grants with performancebased vesting provisions", Review of Financial Studies, 23, 10, 3849-3888.

Cai J. and A.M. Vijh, 2005, "Executive stock option valuation in a two state variable framework", Journal of Derivatives, Spring, 9-27.

Carpenter J.N., 1998, "The exercise and valuation of executive stock options", Journal of Financial Economics, 48, 127-158.

Carpenter J.N., Stanton R. and N. Wallace, 2010, "Optimal Exercise of Executive Stock Options and Implications for Firm Cost", Journal of Financial Economics, 98, 315-337.

Carpenter J.N., Stanton R. and N. Wallace, 2012, "Estimation of employee stock option exercise rates and firm cost", Working Paper, UC Berkeley.

Constantinides, G., 1984, "Warrant exercise and bond conversion in competitive markets", Journal of Financial Economics, 371-397.

Conyon M.J., Fernandes N., Ferreira M.A., Matos P., and Murphy K.J., 2011, "The executive compensation controversy: A transatlantic analysis", Fondazione Rodolfo Dd Benedetti Report.

Core J. and Guay W., 2001, "Stock option plans for non-executive employees", Journal of Financial Economics, 61, 253-287.

Cox J.C. and Rubinstein M., 1985, Options Markets, Prentice Hall Inc., Englewood Cliffs, NJ.

Dennis P.J. and Rendleman R.J., 2008, "Valuing Multiple Employee Stock options issued by the same company", Journal of Derivatives, 16(1), 44-69.

Detemple J. and Sundaresan S., 1999, "Nontraded asset valuation with portfolio constraints: a binomial approach", Review of Financial Studies, 12, 835-872.

Frydman C. and Saks R.E., 2010, "Executive compensation: a new view from a long term perspective, 1936-2005”, Review of Financial Studies, 23, 5, 2100-2138. 
Grasselli M. and Henderson V., 2009, "Risk Aversion and Block Exercise of Executive Stock Options", Journal of Economic Dynamics and Control, 33, 109-127.

Hall B.J. and Murphy K.J., 2000, "Optimal Exercise Prices for Executive Stock Options", American Economic Review Papers and Proceedings, 90(2), 209-214.

Hall B.J. and Murphy K.J., 2002, "Stock options for undiversified employees", Journal of Accounting and Economics, 33, 3-42.

Heath C. Huddart S. and Lang M., 1999, "Psychological factors and stock option exercise", Quarterly Journal of Economics, 114(2), 601-627.

Henderson V., 2005, "The impact of the market portfolio on the valuation, incentives and optimality of executive stock options", Quantitative Finance, 5(1), 35-47.

Henderson V. and Hobson D., 2011, "Optimal Liquidation of Derivative Portfolios", Mathematical Finance, 21, 3, 365-382

Henderson V., Sun J. and Whalley, A.E., 2012, "Portfolios of American Options under General Preferences: Results and Counterexamples", forthcoming Mathematical Finance. Earlier version available at: SSRN: http://ssrn.com/abstract=1769673.

Huddart S, 1994, "Employee stock options", Journal of Accounting and Economics, 18, 207-231.

Huddart S. and Lang M., 1996, "Employee stock option exercises: an empirical analysis", Journal of Accounting and Economics, 21, 5-43.

Ingersoll J.E., 2006, "The subjective and objective valuation of incentive stock options", Journal of Business, 79, 453-487.

Jain A. and Subramanian A., 2004, "The intertemporal exercise and valuation of employee stock options", The Accounting Review, 79, 3, 705-743.

Johnson, S. A. and Y. S. Tian, 2000, "The Value and Incentive Effects of Nontraditional Executive Stock Option Plans", Journal of Financial Economics, 57, 3-34.

Kahl M., Liu J. and Longstaff F.A., 2003, "Paper millionaires: how valuable is stock to a stockholder who is restricted from selling it?", Journal of Financial Economics, 67, 385-410.

Klein D. and E. Maug, 2010, "How do executives exercise their stock options?", ECGI Working paper No. 284/2010.

Kole, S.R., 1997, "The complexity of compensation contracts", Journal of Financial Economics, 43, 79-104.

Kulatilaka N. and Marcus A.J., 1994, "Early exercise and the valuation of employee stock options", Financial Analysts Journal, 50, 46-56. 
Lambert R., Larcker D. and Verrechia, R.E., 1991, "Portfolio considerations in valuing executive compensation", Journal of Accounting Research, 29, 129-149.

Leung T. and R. Sircar, 2009, "Accounting for risk aversion, vesting, job termination risk and multiple exercises in valuation of employee stock options", Mathematical Finance, 19, 99-128.

Malemendier U. and G. Tate, 2005, "CEO Overconfidence and Corporate Investment", Journal of Finance, 60, 2661-2700.

Merton R.C., 1971, "Optimum consumption and portfolio rules in a continuous time model", Journal of Economic Theory, 3, 373-413.

Ofek E. and Yermack D., 2000, "Taking stock: equity-based compensation and the evolution of managerial ownership", Journal of Finance, 55, 1367-1384.

Rogers L.C.G.R and Scheinkman J., 2007, "Optimal exercise of executive stock options, Finance and Stochastics, 11, 357-372.

Spatt C.S. and Sterbenz F.P., 1988, "Warrant Exercise, Dividends and Reinvestment Policy", Journal of Finance, 43, 2, 493-506.

Srivastava A., 2011, "The early exercise of executive stock options and firm's future systematic and idiosyncratic risks", Working paper, Kellogg School of Management, Northwestern University.

Sun J., 2011, "Models of Executive Stock Options", PhD Thesis, Warwick Business School, University of Warwick.

Whalley A.E., 2008, "Effect of executive share ownership and private hedging on executive stock option exercise and values", Working paper, Warwick Business School.

Wilmott P., Howison S. and Dewynne J., 1995, "The Mathematics of Financial Derivatives", Cambridge University Press. 


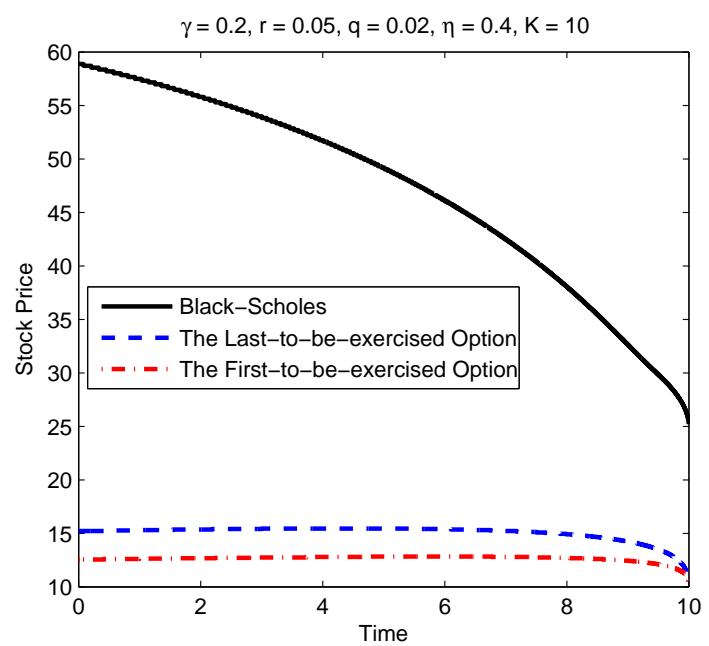

Figure 1: Portfolio of Two identical options with $K_{Y}=10, T_{Y}=5$. Parameter values: $\gamma=0.2, r=0.05, q=0.02, \eta=0.4, \rho=0$. 

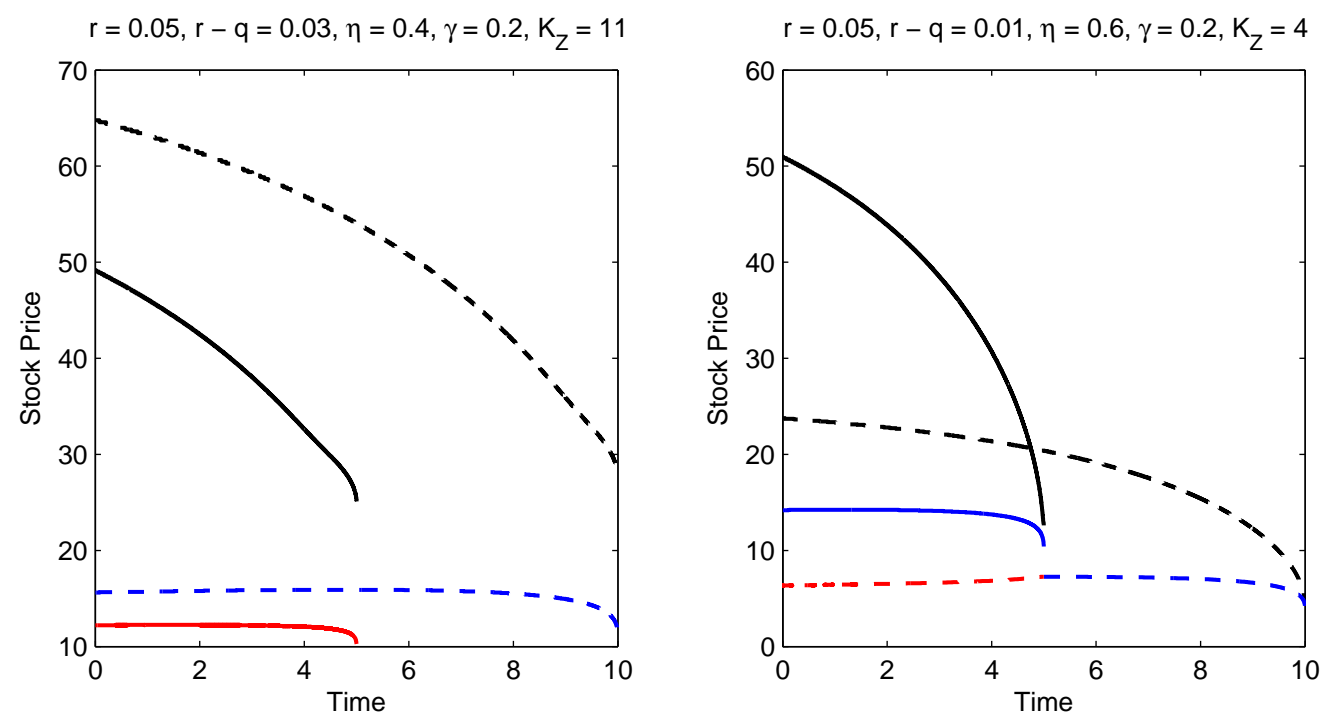

Figure 2: Option $Y$ has $K_{Y}=10, T_{Y}=5$; option $Z$ has $T_{Z}=10$. In the left panel, $K_{Z}=11$; in the right panel $K_{Z}=4$. Parameter values where not stated: $\rho=0$.

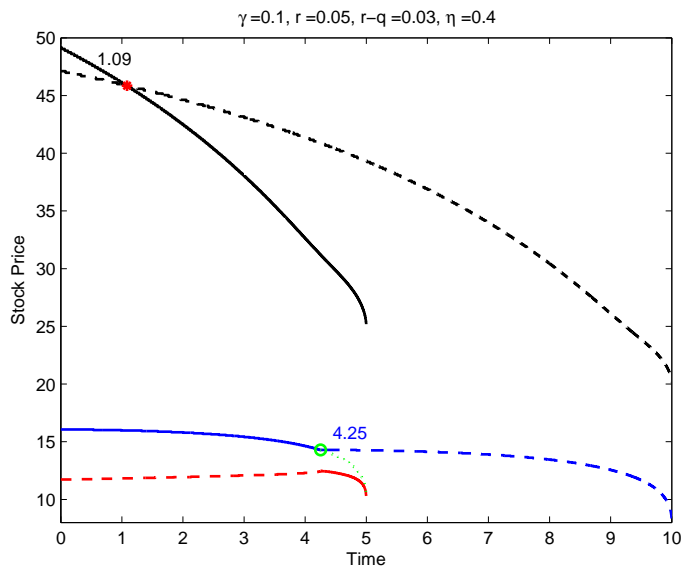

Figure 3: Option $Y$ has $K_{Y}=10, T_{Y}=5$; option $Z$ has $K_{Z}=$ $8, T_{Z}=10$. Parameter values: $\gamma=0.1, r=0.05, q=0.02, \eta=0.4$, $\rho=0$ 


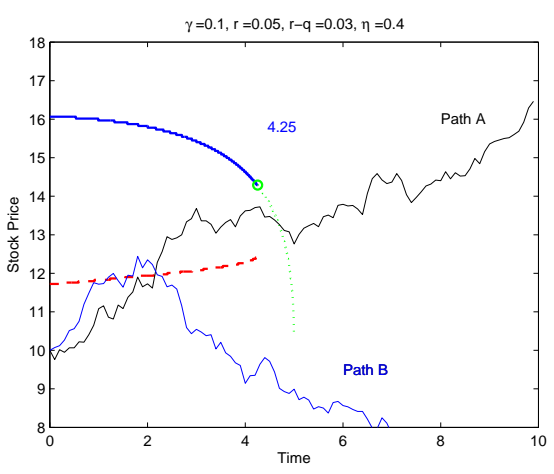

(a)

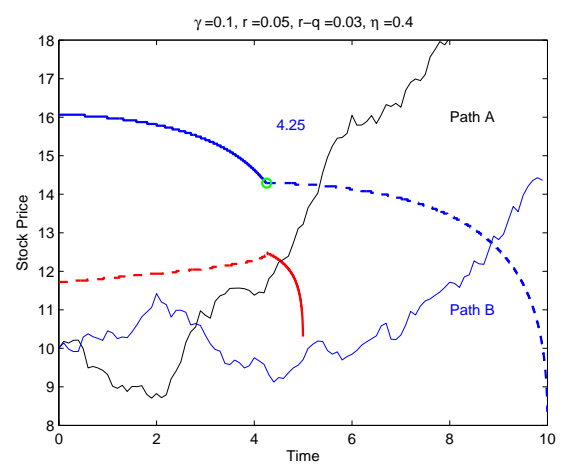

(b)

Figure 4: Option $Y$ has $K_{Y}=10, T_{Y}=5$; option $Z$ has $K_{Z}=8, T_{Y}=10$. Parameter values: $\gamma=0.1, r=0.05, q=0.02, \eta=0.4, \rho=0$.

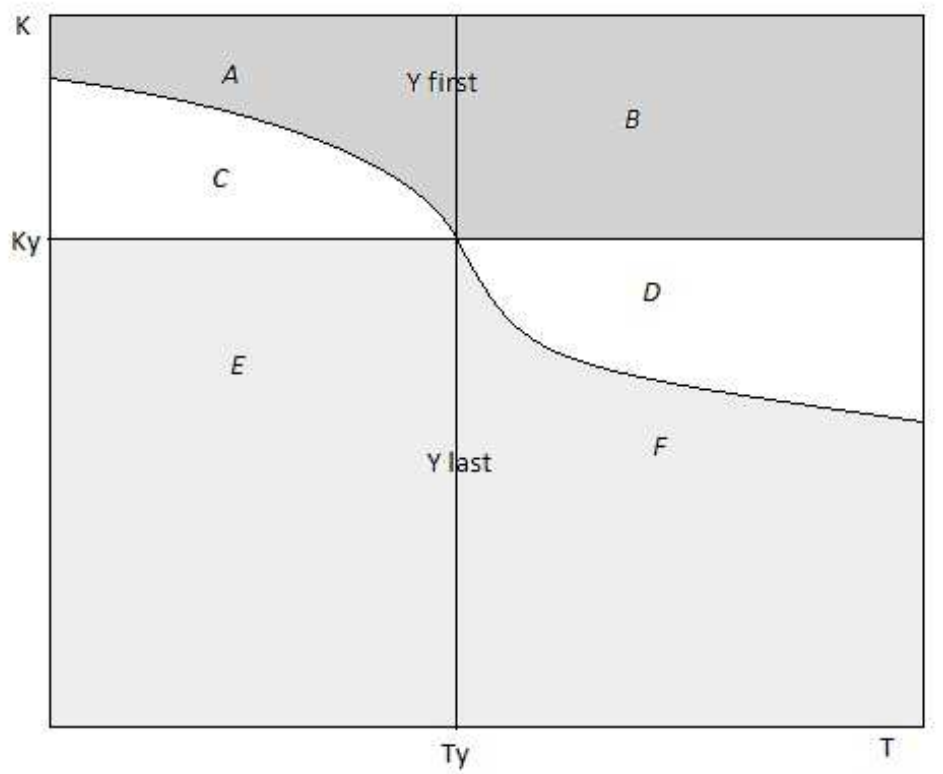

Figure 5: Stylized representation of strike/maturity combinations giving various exercise ordering strategies for base option $Y$ with strike $K_{Y}$, maturity $T_{Y}$ and a second option with a varying strike $K$ and maturity $T$. 


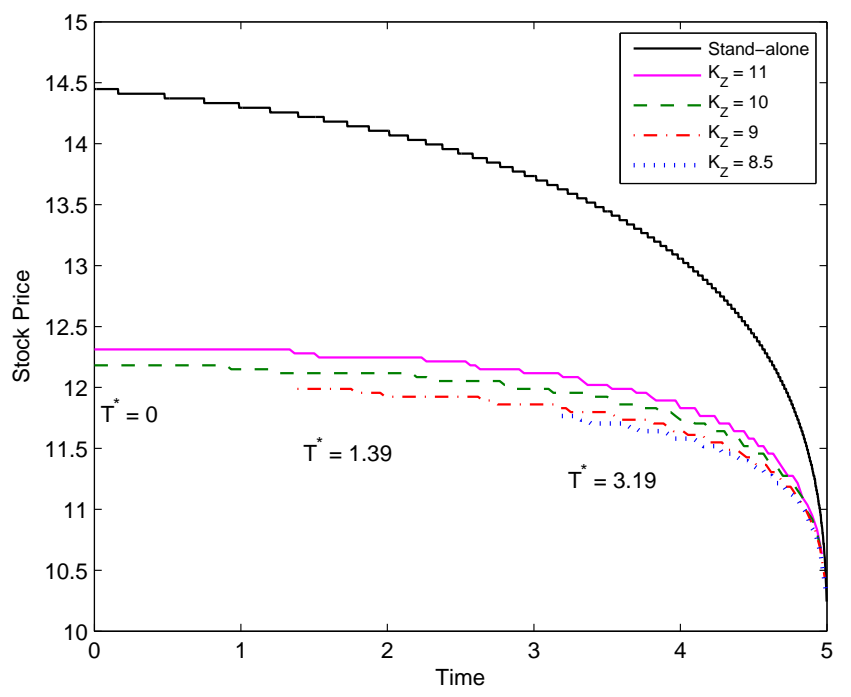

Figure 6: Impact of $K_{Z}$ on thresholds of Option $Y\left(K_{Y}=10, T_{Y}=5\right.$, $\left.T_{Z}=10\right)$. Parameter values: $\gamma=0.2, r=0.05, q=0, \eta=0.2$, $\rho=0$. 


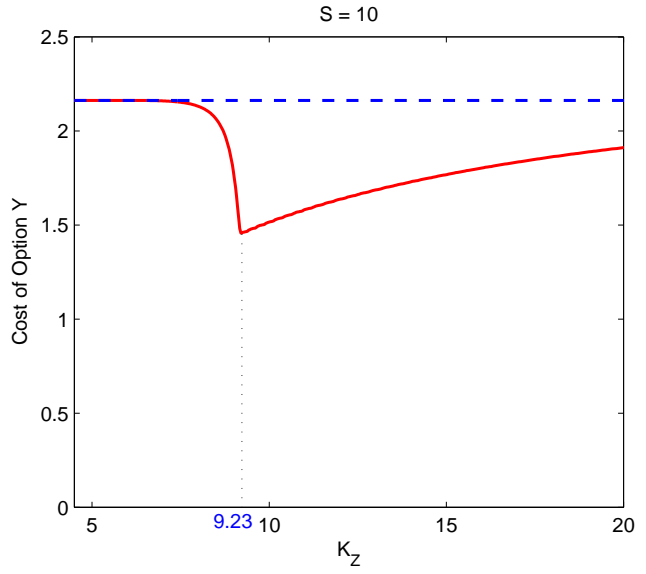

(a)

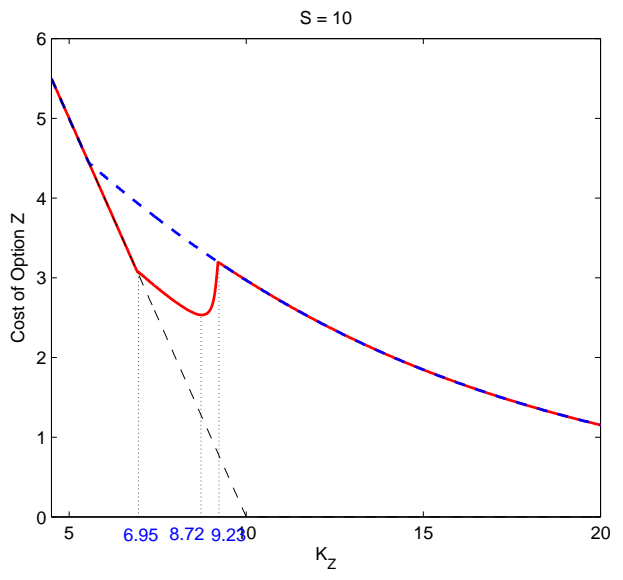

(c)

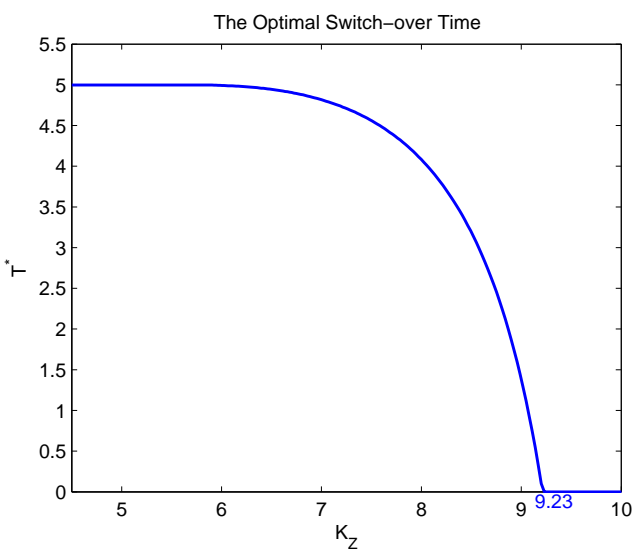

(b)

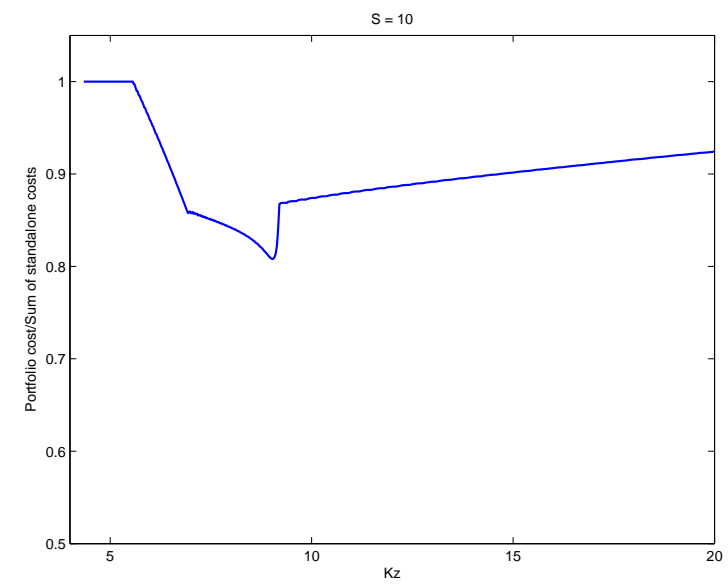

(d)

Figure 7: Top left graph: Cost of option Y, Top right graph: Switchover time, T*, Bottom left graph: Cost of option Z, Bottom right graph: Ratio of cost of portfolio to sum of stand-alone costs; all vs strike price of option $Z$. Option $Y$ has $K_{Y}=10, T_{Y}=5$; option $Z$ has $T_{Z}=10$. Parameter values: $\gamma=0.2, r=0.05, q=0, \eta=0.2, \rho=0$. 


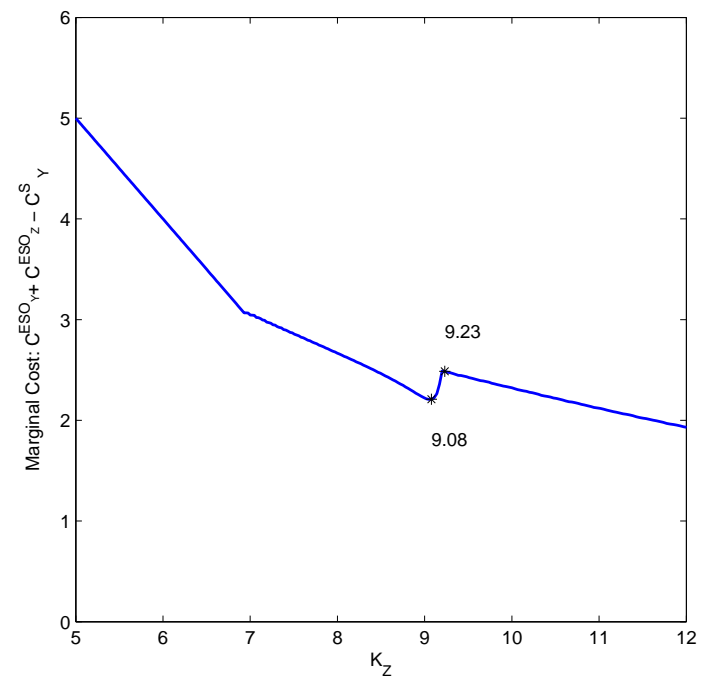

Figure 8: Incremental cost of new grant $Z$ in portfolio with existing ATM option $Y$, as a function of $K_{Z}$. Option $Y$ has $K_{Y}=10$, $T_{Y}=5$. Parameter values: $\gamma=0.2, r=0.05, q=0, \eta=0.2, \rho=0$.

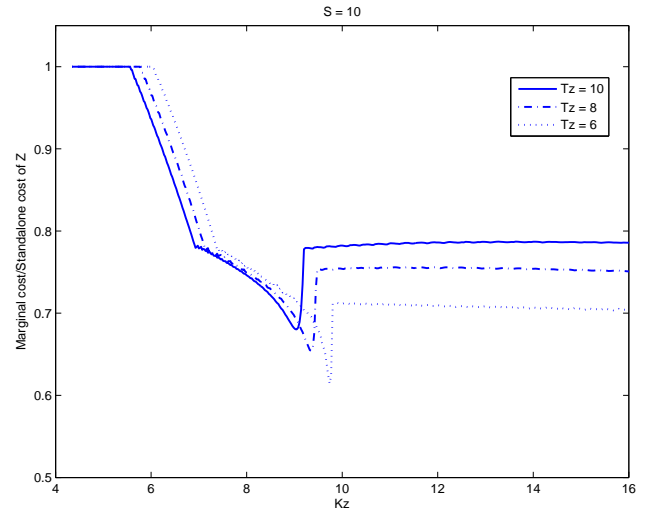

(a)

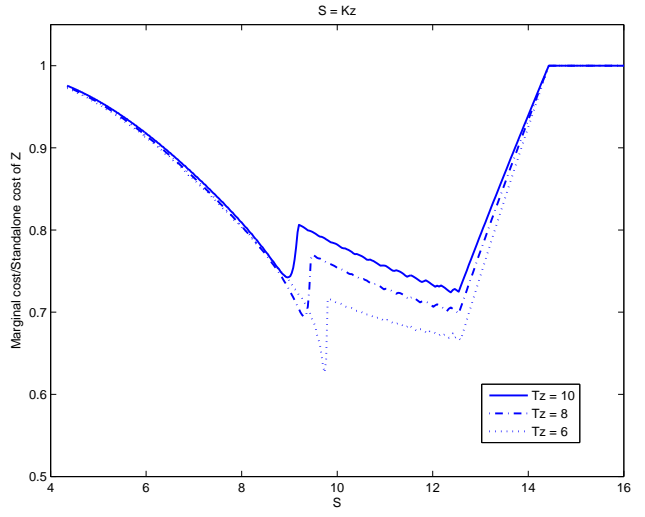

(b)

Figure 9: Ratio of incremental cost of new grant $Z$ in portfolio with existing option $Y$, as a proportion of stand-alone cost of $Z$ for different $T_{Z} s$. Left graph vs $K_{Z}$ with $S=10$, right graph vs $S$ for ATM option grant of $Z$. Option $Y$ has $K_{Y}=10, T_{Y}=5$. Parameter values: $\gamma=0.2$, $r=0.05, q=0, \eta=0.2, \rho=0$. 


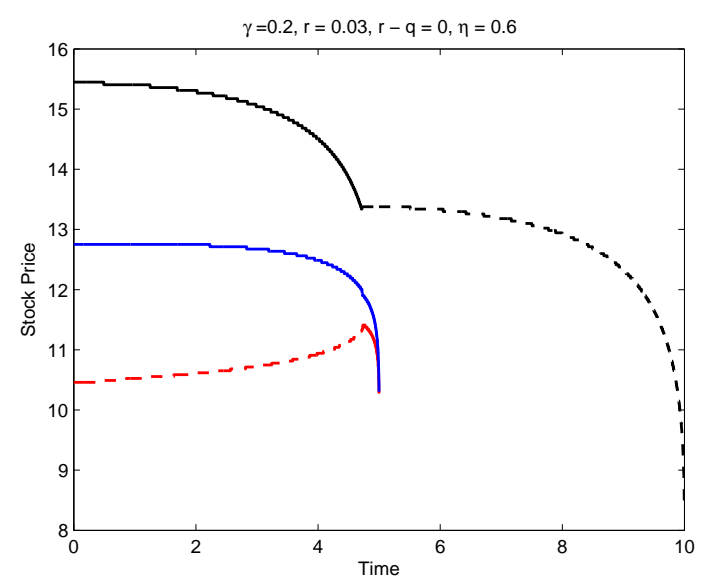

Figure 10: Portfolio with Two options with $K_{Y}=10, T_{Y}=5$ and one option with $K_{Z}=8, T_{Z}=10$. Parameter values: $\gamma=0.2, r=0.03$, $q=0.03, \eta=0.6, \rho=0$. 